\title{
MEAN CONVERGENCE OF LAGRANGE INTERPOLATION. III
}

\author{
BY \\ PAUL NEVAI ${ }^{1}$
}

\begin{abstract}
Necessary and sufficient conditions are found for weighted mean convergence of Lagrange and quasi-Lagrange interpolation based at the zeros of generalized Jacobi polynomials.
\end{abstract}

1. Introduction. Although the problem of finding necessary and sufficient conditions for weighted mean convergence of Lagrange interpolation based at the zeros of orthogonal polynomials was formulated over forty years ago by P. Erdös and P. Turán, nevertheless no significant progress was achieved until 1970 when R. Askey [1 and 2] partially solved the case of interpolating at zeros of Jacobi polynomials. Askey's approach was based on an idea dating back to J. Marcinkiewicz which consists of reducing the problem of mean convergence of Lagrange interpolation to that of orthogonal Fourier series. In order to accomplish this task certain quadrature sums had to be estimated in terms of integrals. This was successfully carried out by Askey for certain Jacobi weights. By proposing another approach to estimating quadrature sums and by refining Askey's techniques, I managed to improve Askey's results in [14] where I found sufficient conditions for weighted $L^{p}(0<p<\infty)$ convergence of Lagrange interpolation based at the zeros of slightly generalized Jacobi polynomials. By solving Turán's problem in [13, p. 180] I showed that for $0<p<\infty$ the conditions given in [14] are necessary as well. The only limitation of the Fourier series method is that it requires the knowledge of convergence of Fourier series in the same weighted $L^{p}$ space where the convergence of Lagrange interpolation is considered. Since at the present time nothing is known about convergence of orthogonal Fourier series in $L^{p}$ spaces with arbitrary weights, one is forced to look for other approaches when considering convergence of Lagrange interpolation in $L^{p}$ spaces with general weights. It is demonstrated in this paper that by realizing that, in fact, Lagrange interpolation can be looked at as a mapping from bounded functions into the weighted $L^{p}$ space rather than as a mapping from $L^{p}$ into $L^{p}$, one can directly estimate $L^{p}$ norms without referring to Fourier series. Quadrature sums still need to be handled in a proper way, but that technique was worked out in [13]. In

Received by the editors March 7, 1983. The contents of this paper have been presented at the Special Session on Approximation Theory at the Evanston, Illinois, meeting of the American Mathematical Society on November 11, 1983.

1980 Mathematics Subject Classification. Primary 41A05, 42 C05.

Key nords and phrases. Lagrange interpolation, orthogonal polynomials.

${ }^{1}$ This paper is based upon research supported by the National Science Foundation under Grant No. MCS 81-01720.

"1984 American Mathematical Society $0002-9947 / 84 \$ 1.00+\$ .25$ per page 
this paper we set the goal of finding necessary and sufficient conditions for convergence of Lagrange interpolation based at the zeros of generalized Jacobi polynomials in $L^{p}$ spaces with general weights. In fact, we are going to consider quasi-Lagrange interpolating polynomials which have a property that they interpolate not just at the zeros of orthogonal polynomials but also possibly at two more exceptional points and, at these exceptional points, their derivatives vanish at a prescribed rate. It turns out that although by doing so we might ruin convergence when ordinary Lagrange interpolation does converge, nevertheless these quasiLagrange interpolation polynomials will converge when ordinary Lagrange interpolation does not. This phenomenon is described in Theorem 6 which is the main result of this paper. In case the reader is interested in the history of the problem of mean convergence of Lagrange interpolation, we suggest [1,14 and 15] as references. An application of the results of this paper to weighted mean convergence of HermiteFejér interpolation is given in [16].

\section{Notations, auxiliary results and the Lemma.}

General notations. $\mathbf{N}$ is the set of positive integers. The symbol "const" stands for some positive constant taking a different value each time it is used. It will always be clear what variables and indices the constants are independent of. If $A$ and $B$ are two expressions depending on some variables then we write

$$
A \sim B \quad \text { if }\left|A B^{-1}\right| \leqslant \text { const and }\left|A^{-1} B\right| \leqslant \text { const }
$$

uniformly for the variables in consideration. The characteristic function of a set $\Delta$ is denoted by $1_{\Delta}$. For the sake of brevity we will frequently omit unnecessary parameters. For example, if we have an expression $x_{k n}(w)$ and $w$ does not vary in a given context, then we will write $x_{k n}$ instead of $x_{k n}(w)$, or $\int f$ will stand for $\int f(x) d x$. The integer part of a number $c$ is denoted by $[c]$.

Spaces of functions. We define $L^{p},\left(L \log ^{+} L\right)^{p}$ and $C$ in the usual way. Unless otherwise specified, all these spaces are spaces of real-valued functions with domain in $[-1,1]$. For the sake of convenience we retain the notation $\|\cdot\|_{p}$ even when $0<p<1$. Thus e.g. $f \in\left(L \log ^{+} L\right)^{p}(0<p<\infty)$ if and only if

$$
\left\|f \log ^{+}|f|\right\|_{p}=\left[\int_{-1}^{1}\left\{|f(t)| \log ^{+}|f(t)|\right\}^{p} d t\right]^{1 / p}<\infty .
$$

Hilbert transforms. For $f \in L^{1}$ in $[-1,1]$ the Hilbert transform $H(f)$ is defined by

$$
H(f, x)=\lim _{\varepsilon \rightarrow 0} \int_{|x-t| \geqslant \varepsilon} \frac{f(t)}{x-t} d t .
$$

We are going to use two properties of the Hilbert transform, namely, that $H$ is a bounded operator in $L^{p}$ for $1<p<\infty$ (M. Riesz) and

$$
\int H(f) g=-\int f H(g)
$$

whenever $f \in L^{p}$ and $g \in L^{q}, p^{-1}+q^{-1}=1$. (See [4, pp. 1059-1060].)

Orthogonal polynomials. If $w$ is a nonnegative $L^{1}$ function supported in $[-1,1]$ such that $\|w\|_{1}>0$, then the corresponding system of orthogonal polynomials is denoted 
by $\left\{p_{n}(w)\right\}_{0}^{\infty}$. Hence

$$
p_{n}(w, x)=\gamma_{n}(w) x^{n}+\text { lower degree terms, } \gamma_{n}(w)>0,
$$

and

$$
\int_{-1}^{1} p_{n}(w) p_{m}(w) w=\delta_{n m}
$$

The zeros of $p_{n}(w)$ are denoted by $x_{k n}(w)$ and they are ordered so that

$$
x_{1 n}(x)>x_{2 n}(x)>\cdots>x_{n n}(w) .
$$

The reproducing kernel $K_{n}(w)$ is defined by

$$
K_{n}(w, x, t)=\sum_{k=0}^{n-1} p_{k}(w, x) p_{k}(w, t)
$$

which by the Christoffel-Darboux formula [18, p. 43] is equivalent to

$$
K_{n}(w, x, t)=\frac{\gamma_{n-1}(w)}{\gamma_{n}(w)} \frac{p_{n}(w, x) p_{n-1}(w, t)-p_{n-1}(w, x) p_{n}(w, t)}{x-t}
$$

The Christoffel function $\lambda_{n}(w)$ is defined by $\lambda_{n}(w, x)=K_{n}(w, x, x)^{-1}$. The numbers

$$
\lambda_{k n}(w)=\lambda_{n}\left(w, x_{k n}(w)\right), \quad 1 \leqslant k \leqslant n,
$$

are the Cotes numbers and they appear in the Gauss-Jacobi quadrature formula [18, p. 47]

$$
\sum_{k=1}^{n} R\left(x_{k n}(w)\right) \lambda_{k n}(w)=\int_{-1}^{1} R w
$$

which is valid for every polynomial $R$ of degree less than $2 n$. Since

$$
\frac{\gamma_{n-1}(w)}{\gamma_{n}(w)}=\int_{-\infty}^{\infty} t p_{n-1}(w, t) p_{n}(w, t) w(t) d t
$$

and $w$ is supported in $[-1,1]$ we have

$$
\gamma_{n-1}(w) / \gamma_{n}(w) \leqslant 1
$$

for $n \in \mathbf{N}$.

Lagrange interpolation. For a given weight $w$ and bounded function $f$ the corresponding Lagrange interpolating polynomial is denoted by $L_{n}(w, f)$. Thus

$$
L_{n}\left(w, f, x_{k n}(w)\right)=f\left(x_{k n}(w)\right), \quad 1 \leqslant k, \leqslant n, n \in \mathbf{N},
$$

and we can write

$$
L_{n}(w, f)=\sum_{k=1}^{n} f\left(x_{k n}(w)\right) l_{k n}(w)
$$

where the fundamental polynomials $l_{k n}(w)$ are defined by

$$
l_{k n}(w, x)=\frac{p_{n}(w, x)}{p_{n}^{\prime}\left(w, x_{k n}(w)\right)\left(x-x_{k n}(w)\right)}, \quad 1 \leqslant k \leqslant n
$$


Another expression for $l_{k n}(w)$ is

$$
l_{k n}(w, x)=\frac{\gamma_{n-1}(w)}{\gamma_{n}(w)} \lambda_{k n}(w) p_{n-1}\left(w, x_{k n}(w)\right) \frac{p_{n}(w, x)}{x-x_{k n}(w)}
$$

(see [18, p. 48]). If $w$ is supported in $[-1,1]$ then all the zeros $x_{k n}(w)$ of $p_{n}(w)$ belong to $(-1,1)[18$, p. 44] so that $(2)$ does make sense even if $f$ is only bounded on every closed subinterval of $(-1,1)$ but not on $[-1,1]$. We will need the identity [7, p. 25]

$$
\lambda_{n}(w, x)^{-1}=\sum_{k=1}^{n} \lambda_{k n}(w)^{-1} l_{k n}(w, x)^{2} .
$$

Quasi-Lagrange interpolation. Let $w$ be a weight function with support in $[-1,1]$ and let $r$ and $s$ be nonnegative integers. If $f$ is a bounded function in $[-1,1]$ then the quasi-Lagrange interpolating polynomial $L_{n}^{(r, s)}(w, f)$ is the unique polynomial of degree at most $n+r+s-1$ which satisfies

$$
L_{n}^{(r . s)}\left(w, f, x_{k n}(w)\right)=f\left(x_{k n}(w)\right), \quad 0 \leqslant k \leqslant n+1,
$$

where $x_{0 n}(w)=1$ and $x_{n+1, n}(w)=-1$,

$$
L_{n}^{(r, s)}(w, f, 1)^{(l)}=0, \quad 1 \leqslant l \leqslant r-1,
$$

and

$$
L_{n}^{(r . s)}(w, f,-1)^{(l)}=0, \quad 1 \leqslant l \leqslant s-1 .
$$

If either $r$ or $s$ equals 0 then $k=0$ or $k=n+1$, respectively, is omitted in (4). Naturally, $L_{n}^{(r . s)}(w, f)$ is a Hermite interpolating polynomial, and it can explicitly be represented in the form

$$
L_{n}^{(r, s)}(w, f)=\sum_{k=0}^{n+1} f\left(x_{k n}(w)\right) h_{k n}(w)
$$

where

$$
\begin{array}{r}
h_{k n}(w, x)=(1-x)^{r}(1+x)^{s}\left(1-x_{k n}(w)\right)^{-r}\left(1+x_{k n}(w)\right)^{-s} l_{k n}(w, x) \\
\text { for } 1 \leqslant k \leqslant n
\end{array}
$$

$$
h_{0 n}(w, x)=\left.(1+x)^{s} p_{n}(w, x) \sum_{l=0}^{r-1}(-1)^{l} \frac{1}{l !}\left[\frac{1}{p_{n}(w, t)(1+t)^{s}}\right]^{(l)}\right|_{t=1}(1-x)^{l}
$$

and

(8) $h_{n+1, n}(w, x)=\left.(1-x)^{r} p_{n}(w, x) \sum_{l=0}^{s-1} \frac{1}{l !}\left[\frac{1}{p_{n}(w, t)(1-t)^{r}}\right]^{(l)}\right|_{t=-1}(1+x)^{l}$.

For $r=s=1$ these polynomials have been investigated in [8, 10, 19 and 20].

Jacobi weights. The function $v$ is called a Jacobi weight function if $v$ can be written in the form

$$
v(x)=(1-x)^{a}(1+x)^{b}
$$


for $-1 \leqslant x \leqslant 1$ and $v(x)=0$ for $|x|>1$. In this paper we do not necessarily assume that $v$ is integrable. The function $v$ is a rational Jacobi weight if $a$ and $b$ in (9) are integers.

Generalized Jacobi polynomials. Let $w$ be a nonnegative integrable function defined in $[-1,1]$. We say that $w$ is a generalized Jacobi weight function $(w \in G J)$ if $w$ can be written in form

$$
w(x)=\psi(x)(1-x)^{\Gamma_{0}} \prod_{k=1}^{m}\left|t_{k}-x\right|^{\Gamma_{k}}(1+x)^{\Gamma_{m+1}}
$$

for $-1 \leqslant x \leqslant 1$ where $-1<t_{m}<t_{m-1}<\cdots<t_{1}<1, \Gamma_{k}>-1(k=0,1, \ldots, m+1)$ and $\psi^{ \pm 1} \in L^{\infty}$ in $[-1,1]$. If, in addition, $\psi$ in (10) is continuous and the modulus of continuity $\omega$ of $\psi$ satisfies

$$
\int_{0}^{1} \frac{\omega(t)}{t} d t<\infty
$$

then we say that $w$ is a generalized smooth Jacobi weight $(w \in G S J)$. Orthogonal polynomials corresponding to generalized Jacobi weights are called generalized Jacobi polynomials. Such polynomials and their characteristics have extensively been studied by Badkov [3] and myself [13]. For the convenience of the reader, we provide a collection of properties of generalized Jacobi polynomials which will be applied when investigating mean convergence of Lagrange interpolation.

(a) Let $w \in G J$ and set $x_{k n}(w)=\cos \theta_{k n}$ for $0 \leqslant k \leqslant n+1$ where $x_{0 n}=1$, $x_{n+1, n}=-1$ and $0 \leqslant \theta_{k n} \leqslant \Pi$. Then

$$
\theta_{k+1, n}-\theta_{k, n} \sim 1 / n
$$

uniformly for $0 \leqslant k \leqslant n, n \in \mathbf{N}$. (See [13, Theorem 9.22, p. 166].)

(b) Let $w \in G J$ and let $w$ be given by (10). Define $\bar{w}_{n}$ by

$$
\bar{w}_{n}(x)=\left(\sqrt{1-x}+\frac{1}{n}\right)^{2 \Gamma_{0}+1} \prod_{k=1}^{m}\left(\left|t_{k}-x\right|+\frac{1}{n}\right)^{\Gamma_{k}}\left(\sqrt{1+x}+\frac{1}{n}\right)^{2 \Gamma_{m} \cdot 1+1} .
$$

Then

$$
\lambda_{n}(w, x) \sim \frac{1}{n} \bar{w}_{n}(x)
$$

uniformly for $-1 \leqslant x \leqslant 1$ and $n \in \mathbf{N}$ (see [13, Theorem 6.3.28, p. 120]), in particular,

$$
\lambda_{k n}(w) \sim \frac{1}{n}\left(1-x_{k n}(w)\right)^{\Gamma_{0}+1 / 2} \prod_{l=1}^{m}\left(\left|t_{l}-x_{k n}(w)\right|+\frac{1}{n}\right)^{\Gamma_{l}}\left(1+x_{k n}(w)\right)^{\Gamma_{m+1}+1 / 2}
$$

uniformly for $1 \leqslant k \leqslant n, n \in \mathbf{N}$. (Formula (14) follows from (11) and (13).)

(c) If $w \in G S J$ is given by (10) and $\bar{w}_{n}$ is defined by (12) then

$$
\left|p_{n}(w, x)\right| \leqslant \text { const } \bar{w}_{n}(x)^{-1 / 2}
$$


uniformly for $-1 \leqslant x \leqslant 1$ and $n \in \mathbf{N}$ (see [3, Theorem 1.1, p. 226]), in particular,

$$
\left|p_{n}(w, x)\right| \leqslant \text { const }\left[1 / \sqrt{w(x) \sqrt{1-x^{2}}}+1\right]
$$

uniformly for $-1 \leqslant x \leqslant 1, n \in \mathbf{N}$. Moreover,

$$
p_{n}(w, 1) \sim n^{\Gamma_{0}+1 / 2}
$$

and

$$
\left|p_{n}(w,-1)\right| \sim n^{\Gamma_{m+1}+1 / 2}
$$

uniformly for $n \in \mathbf{N}$. (See [13, Corollary 9.34, p. 171].)

(d) If $w$ is a weight function supported in $[-1,1]$ and $\tilde{w}$ is defined by $\tilde{w}(x)=$ $\left(1-x^{2}\right) w(x)$ then

$$
\left(1-x_{k n}(w)^{2}\right) p_{n-1}\left(\tilde{w}, x_{k n}(w)\right)=a_{n} p_{n-1}\left(w, x_{k n}(w)\right), \quad a_{n}>0,
$$

for $1 \leqslant k \leqslant n$ where $a_{n}$ is independent of $k$. If $\log w(\cos \theta)$ is integrable then $\lim _{n \rightarrow \infty} a_{n}=1$ and thus

$$
p_{n-1}\left(w, x_{k n}(w)\right) \sim\left(1-x_{k n}(w)^{2}\right) p_{n-1}\left(\tilde{w}, x_{k n}(w)\right)
$$

uniformly for $1 \leqslant k \leqslant n$ and $n \in \mathbf{N}$. (See [13, Lemma 9.30, p. 170].) If $w \in G S J$ is given by (10) and $\bar{w}_{n}$ is defined by (12) then

$$
\bar{w}_{n}\left(x_{k n}(w)\right) p_{n-1}\left(w, x_{k n}(w)\right)^{2} \sim\left(1-x_{k n}(w)^{2}\right)
$$

uniformly for $1 \leqslant k \leqslant n, n \in \mathbf{N}$. (See [13, Theorem 9.31, p. 170].)

(e) If $w$ is a weight function supported in $[-1,1]$ such that $\log w(\cos \theta)$ is integrable then

$$
0<\lim _{n \rightarrow \infty} 2^{-n} \gamma_{n}(w)<\infty
$$

(See [18, p. 309].)

(f) If $w \in G J$ and $l$ is a positive integer then

$$
\left|p_{n}(w, \pm 1)^{(l)}\right| \leqslant \text { const } n^{2 l}\left|p_{n}(w, \pm 1)\right|
$$

and

$$
\left(1 / p_{n}(w, \pm 1)\right)^{(l)} \mid \leqslant \text { const } n^{2 l}\left|p_{n}(w, \pm 1)\right|^{-1}
$$

uniformly for $n \in \mathbf{N}$. We can prove (22) and (23) by induction. When $l=1$ then

$$
p_{n}( \pm 1)^{\prime}=p_{n}( \pm 1) \sum_{k=1}^{n}\left( \pm 1-x_{k n}\right)^{-1}
$$

and by (11) inequality (22) holds. Otherwise we write

$$
p_{n}(x)^{\prime}=p_{n}(x) \sum_{k=1}^{n}\left(x-x_{k n}\right)^{-1}
$$

and differentiating this identity $l-1$ times we obtain

$$
p_{n}( \pm 1)^{(l)}=\sum_{j=0}^{l-1}\left(\begin{array}{c}
l-1 \\
j
\end{array}\right) p_{n}( \pm 1)^{(j)}(-1)^{l-j-1}(l-1-j) ! \sum_{k=1}^{n}\left( \pm 1-x_{k n}\right)^{j-l}
$$


and by (11)

$$
\left|p_{n}( \pm 1)^{(l)}\right| \leqslant \text { const } n^{2 l} \sum_{j=0}^{l-1}\left|p_{n}( \pm 1)^{(j)}\right| n^{-2 j}
$$

so that (22) follows by induction. When $l=1$ then

$$
\left(\frac{1}{p_{n}( \pm 1)}\right)^{\prime}=\frac{p_{n}( \pm 1)^{\prime}}{p_{n}( \pm 1)^{2}}=-\frac{1}{p_{n}( \pm 1)} \sum_{k=1}^{n}\left( \pm 1-x_{k n}\right)^{-1} \text {. }
$$

Thus by (11) inequality (23) holds. For $l>1$ we use the identity

$$
-p_{n}( \pm 1)\left(\frac{1}{p_{n}( \pm 1)}\right)^{(l)}=\sum_{j=0}^{l-1}\left(\begin{array}{l}
l \\
j
\end{array}\right)\left(\frac{1}{p_{n}( \pm 1)}\right)^{(j)} p_{n}( \pm 1)^{(l-j)}
$$

so that by (22)

$$
\left|\left(\frac{1}{p_{n}( \pm 1)}\right)^{(l)}\right| \leqslant \text { const } n^{2 l} \sum_{j=0}^{l-1}\left|\left(\frac{1}{p_{n}( \pm 1)}\right)^{(j)}\right| n^{-2 j}
$$

and (23) follows by induction.

(g) Let $w \in G J$ and $0<p<\infty$. If $c$ is a fixed positive number and $v$ is an arbitrary, not necessarily integrable, Jacobi weight, then for every polynomial $R$ of degree at most $c n$

$$
\sum_{k=1}^{n}\left|R\left(x_{k n}(w)\right)\right|^{p} v\left(x_{k n}(w)\right) \lambda_{k n}(w) \leqslant \mathrm{const} \int_{-1}^{1}|R(t)|^{p} v(t) w(t) d t .
$$

(See [13, Theorem 9.25, p. 168].)

(h) Let $w \in G J$ and $0<p<\infty$. If $w$ is given by (10) then for any fixed $c>0$ we define $\Delta_{n}^{*}(c)$ by

$$
\Delta_{n}^{*}(c)=\left[-1+c n^{-2}, 1-c n^{-2}\right] \backslash \bigcup_{k=1}^{m}\left[t_{k}-c n^{-1}, t_{k}+c n^{-1}\right] .
$$

Then there exists a $\bar{c}>0$ such that for every polynomial $R$ of degree at most $n$

$$
\left\||R|^{p} w\right\|_{1} \leqslant \text { const }\left\||R|^{p} \cdot w 1_{n}^{\bar{c}}\right\|_{1}
$$

where $1_{n}^{c}$ denotes the characteristic function of $\Delta_{n}^{*}(c)$. (See Theorem 6.3.28 and Remark 6.3.29 in [13, p. 120].)

(i) If $R$ is any polynomial of degree at most $n$ then

$$
\max _{|x| \leqslant 1}|R(x)| \leqslant 12 \max _{|x| \leqslant 1-n^{-2}}|R(x)|, \quad n=2,3, \ldots
$$

This follows immediately from Chebyshev's inequality [12, p. 51] and from the easily verifiable estimate

$$
T_{n}\left(\left(1-n^{-2}\right)^{-1}\right) \leqslant 12, \quad n=2,3, \ldots,
$$

which holds for the Chebyshev polynomial $T_{n}$.

The Lemma. The following proposition is the key to proving weighted $L^{p}$ convergence of Lagrange interpolation. 
Lemma. Let $1<p<\infty, \phi \in G J$ and let $\Omega$ denote the class of functions $G$ such that $|G(x)| \leqslant 1$ almost everywhere in $[-1,1]$ and $G(x)=0$ for $x \notin[-1,1]$. If the function $g$ satisfies $g \phi^{-1} \in L^{p}$ and $g \in L^{p}\left(\log ^{+} L\right)^{p}$ then

$$
\sup _{G \in \Omega}\left\|g \phi^{-1} H(\phi G)\right\|_{p} \leqslant \text { const }\left[1+\left\|g\left(1+\phi^{-1}+\log ^{+}|g|\right)\right\|_{p}\right]
$$

where the constant is independent of $g$.

Proof. First let $\phi \sim 1$ in $[-1,1]$. If

$$
m=\underset{|x| \leqslant 1}{\operatorname{essinf}} \phi(x) \text { and } M=\underset{|x| \leqslant 1}{\operatorname{ess} \operatorname{supp}} \phi(x)
$$

then

$$
\sup _{G \in \Omega}\left\|g \phi^{-1} H(\phi G)\right\|_{p} \leqslant m^{-1} M \sup _{G \in \Omega}\|g H(G)\|_{p} .
$$

Let $\gamma$ denote the constant in Riesz's inequality [17, p. 48]

$$
\|H(G)\|_{q} \leqslant \gamma q\|G\|_{q}
$$

which is valid for $p \leqslant q<\infty$. In particular, if $G \in \Omega$ then (29) implies

$$
\|H(G)\|_{q} \leqslant \gamma q 2^{1 / q}, \quad p \leqslant q<\infty .
$$

Let $\lambda=2^{-1} \gamma^{-1} p^{-1} e^{-2}$. Then we can write

$$
\begin{gathered}
\int_{-1}^{1}|g(t) H(G, t)|^{p} d t=\int_{\substack{|g(t)| \leqslant \exp \{\lambda|H(G, t)|\} \\
|t| \leqslant 1}}|g(t) H(G, t)|^{p} d t \\
\quad+\int_{\substack{\log ^{+}|g(t)| \geqslant \lambda|H(G, t)| \\
|t| \leqslant 1}}|g(t) H(G, t)|^{p} d t \\
\leqslant \int_{-1}^{1}|H(G, t)|^{p} \exp \{\lambda p H(G, t)\} d t+\left.\lambda^{-p} \int_{-1}^{1}\left|g(t) \log ^{+}\right| g(t)\right|^{p} d t \\
=\sum_{k=0}^{\infty} \frac{(\lambda p)^{k}}{k !} \int_{-1}^{1}|H(G, t)|^{p+k} d t+\left.\lambda^{-p} \int_{-1}^{1}\left|g(t) \log ^{+}\right| g(t)\right|^{p} d t .
\end{gathered}
$$

Since $k ! \geqslant(k / e)^{k}$, we can apply (30) to obtain

$$
\begin{aligned}
& \int_{-1}^{1}|g(t) H(G, t)|^{p} d t \\
& \quad \leqslant 2 \sum_{k=0}^{\infty}(\lambda p e)^{k}[\gamma(p+k)]^{\rho+k} k^{-k}+\left.\lambda^{-p} \int_{-1}^{1}\left|g(t) \log ^{+}\right| g(t)\right|^{p} d t .
\end{aligned}
$$

Obviously $(p+k)^{p+k}=p^{p}(1+k / p)^{p} \cdot k^{k}(1+p / k)^{k} \leqslant p^{p} e^{k} k^{k} e^{p}$ so that

$$
2 \sum_{k=0}^{\infty}(\lambda p e)^{k}[\gamma(p+k)]^{p+k} k^{-k} \leqslant 2(\gamma p e)^{p} \sum_{k=0}^{\infty}\left(\lambda \gamma p e^{2}\right)^{k}=4(\gamma p e)^{p}
$$

since $\lambda \gamma p e^{2}=2^{-1}$. Thus if $G \in \Omega$ then

$$
\|g H(G)\|_{p} \leqslant 4^{1 / p} \gamma p e+2 \gamma p e^{2}\left\|g \log ^{+}|g|\right\|_{p}
$$


which together with (28) implies (27) when $\phi \sim 1$. Now let $\phi \in G J$ and $\phi$ not $\sim 1$. Then $\phi$ can be written in the form

$$
\phi(x)=\phi_{1}(x) \prod_{k=1}^{l}\left|x-y_{k}\right|^{\Gamma_{k}}
$$

where $-1 \leqslant y_{l}<y_{l-1}<\cdots<y_{1} \leqslant 1, \Gamma_{k}>-1$ and $\Gamma_{k} \neq 0$ for $1 \leqslant k \leqslant l$ and $\phi_{1} \sim 1$. Choose $\left\{a_{k}\right\}_{1}^{l+1}$ such that $y_{k} \in\left(a_{k+1}, a_{k}\right)$ for $1 \leqslant k \leqslant l,\left(a_{k+1}, a_{k}\right) \cap\left(a_{j+1}, a_{j}\right)=\phi$ for $k \neq j$ and $[-1,1] \subset \cup_{k=1}^{l}\left[a_{k+1}, a_{k}\right] \subset[-2,2]$. Let $\varepsilon$ be defined by $\varepsilon$ $=\frac{1}{2} \operatorname{dist}\left[\left\{y_{k}\right\},\left\{a_{k}\right\}\right]$ and let $1_{k}$ denote the characteristic function of $\left[a_{k+1}, a_{k}\right)$. Then we can write

$$
\begin{aligned}
g \phi^{-1} H(\phi G)= & \sum_{k=1}^{l} 1_{k} g \phi^{-1} H\left(\phi G \sum_{j=1}^{l} 1_{j}\right) \\
= & \sum_{k-j \geqslant 2} 1_{k} g \phi^{-1} H\left(\phi G 1_{j}\right)+\sum_{k=2}^{l} 1_{k} g \phi^{-1} H\left(\phi G 1_{k-1}\right) \\
& +\sum_{k=1}^{l-1} 1_{k} g \phi^{-1} H\left(\phi G 1_{k+1}\right)+\sum_{k=1}^{l} 1_{k} g \phi^{-1} H\left(\phi G 1_{k}\right) \\
\equiv & \sum^{1}+\sum^{2}+\Sigma^{3}+\sum^{4} .
\end{aligned}
$$

Assume that $G \in \Omega$. We will estimate each sum $\Sigma^{i}$ in (32) individually. If $|k-j| \geqslant 2$ then

$$
1_{k}(x) H\left(\phi G 1_{j}, x\right)=1_{k}(x) \int_{a_{j+1}}^{a_{j}} \frac{\phi(t) G(t)}{x-t} d t
$$

so that

$$
\left|1_{k} g \phi^{-1} H\left(\phi G 1_{j}\right)\right| \leqslant \frac{1}{\varepsilon}\left|1_{k} g \phi^{-1}\right| \int_{a_{j+1}}^{a_{j}} \phi(t) d t .
$$

Hence we obtain

$$
\left|\Sigma^{1}\right| \leqslant \frac{1}{\varepsilon}\left|g \phi^{-1}\right| \cdot\|\phi\|_{1}
$$

and

$$
\left\|\Sigma^{1}\right\|_{p} \leqslant \frac{1}{\varepsilon}\|\phi\|_{1}\left\|g \phi^{-1}\right\|_{p} .
$$

Now we turn to estimating $\Sigma^{2}$ in (32). If

$$
N=\operatorname{ess} \sup \phi(t), \quad t \in \bigcup_{k=1}^{l+1}\left[a_{k}-\varepsilon, a_{k}+\varepsilon\right],
$$

then from

$$
1_{k} g \phi^{-1} H\left(\phi G 1_{k-1}\right)=1_{k} g \phi^{-1}\left[\int_{a_{k}}^{a_{k}+\varepsilon} \frac{\phi(t) G(t)}{x-t} d t+\int_{a_{k}+\varepsilon}^{a_{k-1}} \frac{\phi(t) G(t)}{x-t} d t\right]
$$


we get

$$
\left|1_{k} g \phi^{-1} H\left(\phi G 1_{k-1}\right)\right| \leqslant\left|1_{k} g \phi^{-1}\right|\left[N \log \frac{5}{\left|a_{k}-x\right|}+\frac{1}{\varepsilon} \int_{a_{k}}^{a_{k-1}} \phi(t) d t\right] .
$$

Let

$$
L=\operatorname{ess} \operatorname{supp} \phi(t)^{-1}, \quad t \in[-1,1] \cap \bigcap_{k=1}^{1+1}\left[a_{k}-\varepsilon, a_{k}+\varepsilon\right] .
$$

Then we obtain from (34)

$$
\begin{aligned}
\left|1_{k} g \phi^{-1} H\left(\phi G 1_{k-1}\right)\right| \leqslant & N\left|1_{k} g \phi^{-1}\right| \log \frac{5}{\left|a_{k}-x\right|} 1_{\left[a_{k+1}, a_{k}-\varepsilon\right]} \\
& +N\left|1_{k} g \phi^{-1}\right| \log \frac{5}{\left|a_{k}-x\right|} 1_{\left[a_{k}-\varepsilon, a_{k}\right]}+\frac{1}{\varepsilon}\left|1_{k} g \phi^{-1}\right| \int_{a_{k}}^{a_{k-1}} \phi(t) d t \\
\leqslant & N \log \frac{5}{\varepsilon}\left|1_{k} g \phi^{-1}\right|+N L\left|1_{k} g\right| \log \frac{5}{\left|a_{k}-x\right|} 1_{\left[a_{k}-\varepsilon, a_{k}\right]} \\
& +\frac{1}{\varepsilon}\left|1_{k} g \phi^{-1}\right| \int_{a_{k}}^{a_{k-1}} \phi(t) d t .
\end{aligned}
$$

Since obviously

$$
\begin{array}{r}
g(x) \log \frac{5}{\left|a_{k}-x\right|} \leqslant 2 p|g(x)| \log ^{+}|g(x)|+\left(\frac{5}{\left|a_{k}-x\right|}\right)^{1 / 2 p} \log \frac{5}{\left|a_{k}-x\right|}, \\
-1 \leqslant x \leqslant 1,
\end{array}
$$

we have the inequality

$$
\begin{aligned}
\left|1_{k} g \phi^{-1} H\left(\phi G 1_{k-1}\right)\right| \leqslant & \left|1_{k} g \phi^{-1}\right|\left[N \log \frac{5}{\varepsilon}+\frac{1}{\varepsilon}\|\phi\|_{1}\right]+2 N L p\left|1_{k} g \log ^{+}\right| g|| \\
& +N L 5^{1 / 2 p} 1_{k}\left|a_{k}-x\right|^{-1 / 2 p} \log \frac{5}{\left|a_{k}-x\right|} .
\end{aligned}
$$

Hence

$$
\begin{aligned}
\left|\Sigma^{2}\right| \leqslant & \left|g \phi^{-1}\right|\left[N \log \frac{5}{\varepsilon}+\frac{1}{\varepsilon}\|\phi\|_{1}\right]+2 N L p|g| \log ^{+}|g| \\
& +N L 5^{1 / 2 p} \sum_{k=1}^{l+1}\left|a_{k}-x\right|^{-1 / 2 p} \log \frac{5}{\left|a_{k}-x\right|} .
\end{aligned}
$$

Observing that the last function on the right-hand side of (35) belongs to $L^{p}$, and denoting its $L^{p}$ norm by $K$, we obtain

$$
\left\|\Sigma^{2}\right\|_{p} \leqslant\left[N \log \frac{5}{\varepsilon}+\frac{1}{\varepsilon}\|\phi\|_{1}\right]\left\|g \phi^{-1}\right\|_{p}+2 N L p\left\|g \log ^{+}|g|\right\|_{p}+K N L 5^{1 / 2 p}
$$

The sum $\Sigma^{3}$ in (32) can be estimated in exactly the same way as $\Sigma^{2}$, and by doing so we get

$$
\left\|\Sigma^{3}\right\|_{p} \leqslant\left[N \log \frac{5}{\varepsilon}+\frac{1}{\varepsilon}\|\phi\|_{1}\right]\left\|g \phi^{-1}\right\|_{p}+2 N L p\left\|g \log ^{+}|g|\right\|_{p}+K N L 5^{1 / 2 p}
$$


Now it remains to estimate $\Sigma^{4}$ in (32). Note that for every $k, 1 \leqslant k \leqslant l$, we can represent $\phi$ in the form $\phi(x)=\psi_{k}(x)\left|x-y_{k}\right|^{\Gamma_{k}}$ where $1_{k} \psi_{h}^{+1} \in L^{\infty}$ in $[-1,1]$. Moreover,

$$
\begin{aligned}
1_{k} g \phi^{-1} H\left(\phi G 1_{k}\right) & =1_{k} g \psi_{k}^{-1} H\left(\psi_{k} G 1_{k}\right)+1_{k} g \psi_{k}^{-1}\left[\psi_{k} \phi^{-1} H\left(\phi G 1_{k}\right)-H\left(\psi_{k} G 1_{k}\right)\right] \\
& =\sigma_{k}^{1}+\sigma_{k}^{2} .
\end{aligned}
$$

Since $1_{k} \psi_{k}^{ \pm 1} \in L^{\infty}$ we can apply (31) to conclude that

$$
\left\|\sigma_{k}^{1}\right\|_{p} \leqslant\left\|1_{k} \psi_{k}^{-1}\right\|_{\infty}\left\|1_{k} \psi_{k}\right\|_{\infty}\left[4^{1 / p} \gamma p e+2 \gamma p e^{2}\left\|g \log ^{+}|g|\right\|_{p}\right]
$$

In order to estimate $\sigma_{k}^{2}$ in (38) we write it as

$$
\sigma_{k}^{2}(x)=1_{k}(x) g(x) \psi_{k}(x)^{-1} \int_{a_{k+1}}^{a_{k}} \psi_{k}(t) G(t)\left[\left|\frac{t-y_{k}}{x-y_{k}}\right|^{\Gamma_{k}}-1\right] \frac{1}{x-t} d t .
$$

Hence

$$
\left|\sigma_{k}^{2}\right| \leqslant\left.\left|1_{k} g \psi_{k}^{-1}\right|\left\|1_{k} \psi_{k}\right\|_{\infty} \int_{a_{k+1}}^{a_{k}}|| \frac{t-y_{k}}{x-y_{k}}\right|^{\Gamma_{k}}-1 \mid \frac{1}{|| x-y_{k}|-| t-y_{k} \|} d t .
$$

Introducing a new variable $u=\left(t-y_{k}\right)\left|x-y_{k}\right|^{-1}$ in the integral and observing that $\left|a_{k}\right| \leqslant 2,\left|y_{k}\right| \leqslant 1$ for $1 \leqslant k \leqslant l$, we obtain

$$
\begin{aligned}
\left.\int_{a_{k+1}}^{a_{k}}|| \frac{t-y_{k}}{x-y_{k}}\right|^{\Gamma_{k}}- & 1\left|\frac{1}{|| x-y_{k}|-| t-y_{k}||} d t \leqslant 2 \int_{0}^{3\left|x-y_{k}\right|^{-1}}\right| u^{\Gamma_{k}}-1|| u-\left.1\right|^{-1} d u \\
& =2 \int_{0}^{3 / 2}\left|u^{\Gamma_{k}}-1\right||u-1|^{-1} d u+2 \int_{3 / 2}^{3\left|x-y_{k}\right|^{-1}}\left|u^{\Gamma_{k}}-1\right||u-1|^{-1} d u \\
& \leqslant 2 \int_{0}^{3 / 2}\left|u^{\Gamma_{k}}-1\right||u-1|^{-1} d u+ \begin{cases}6 \Gamma_{k}^{-1} 3^{\Gamma_{k}}\left|x-y_{k}\right|^{-\Gamma_{k}}, & \Gamma_{k}>0, \\
6 \log \left[3\left|x-y_{k}\right|^{-1}\right], & \Gamma_{k}<0 .\end{cases}
\end{aligned}
$$

Substituting this estimate into (40) we get

$$
\begin{aligned}
\left|\sigma_{k}^{2}\right| \leqslant\left|1_{k} g\right|\left\|1_{k} \psi_{k}^{-1}\right\|_{\infty}\left\|1_{k} \psi_{k}\right\|_{\infty} \cdot 2 \int_{0}^{3 / 2}\left|u^{\Gamma_{k}}-1\right||u-1|^{-1} d u \\
+\left|1_{k} g\right|\left\|1_{k} \psi_{k}\right\|_{\infty}\left\{\begin{array}{l}
6 \Gamma_{k}^{-1} 3^{\Gamma_{k}} \phi^{-1}, \quad \Gamma_{k}>0, \\
6\left\|1_{k} \psi_{k}^{-1}\right\|_{\infty} \log \left[3\left|x-y_{k}\right|^{-1}\right], \quad \Gamma_{k}<0 .
\end{array}\right.
\end{aligned}
$$

If $\Gamma_{k}>0$ then (41) implies

$$
\left\|\sigma_{k}^{2}\right\| p \leqslant \text { const }\left\|g\left(1+\phi^{-1}\right)\right\|_{p}, \quad \Gamma_{k}>0 .
$$

Otherwise we observe that

$$
\begin{aligned}
& |g(x)| \log \left[3\left|x-y_{k}\right|^{-1}\right] \\
& \quad \leqslant 2 p|g(x)| \log ^{+}|g(x)|+\left(3 /\left|x-y_{k}\right|\right)^{1 / 2 p} \log \left[3\left|x-y_{k}\right|^{-1}\right]
\end{aligned}
$$

where the second function on the right-hand side of (43) obviously belongs to $L^{p}$. Thus by (41)

$$
\left\|\sigma_{k}^{2}\right\|_{p} \leqslant \text { const }\left[1+\left\|g\left(1+\log ^{+}|g|\right)\right\|_{p}\right], \quad \Gamma_{k}<0 .
$$


Combining (32), (38), (39), (42) and (44) we obtain

$$
\left\|\Sigma^{4}\right\|_{p} \leqslant \text { const }\left[1+\left\|g\left(1+\phi^{-1}+\log ^{+}|g|\right)\right\|_{p}\right] .
$$

Now (27) follows from (32), (33), (36), (37) and (45). The proof of the Lemma has been completed.

\section{Main results.}

THEOREM 1. Let $w \in G S J$ and $0<p<\infty$. Let $v$ be a not necessarily integrable Jacobi weight function, and let $u$ be a nonnegative function defined in $[-1,1]$ such that $u \in L^{p}, u v \in\left(L \log ^{+} L\right)^{p}, u / \sqrt{w \sqrt{1-x^{2}}} \in L^{p}$ and $v \sqrt{w \sqrt{1-x^{2}}} \in L^{1}$. Then for every bounded function $f$

$$
\sup _{n \geqslant 1}\left\|L_{n}(w, v f) u\right\|_{p} \leqslant \text { const }\|f\|_{\infty}
$$

with some constant independent of $f$.

Proof. First let $1<p<\infty$. Since $w \in G S J$, we can write $w$ in the form

$$
w(x)=\psi(x)(1-x)^{\Gamma_{0}} \prod_{k=1}^{m}\left|t_{k}-x\right|^{\Gamma_{k}}(1+x)^{\Gamma_{m} \cdot 1}, \quad \psi^{ \pm 1} \in L^{x},
$$

where $-1<t_{m}<t_{m-1}<\cdots<t_{1}<1$. Let $\tau$ be the set of all indices $k$ for which $\Gamma_{k}$ in (47) is negative and $1 \leqslant k \leqslant m$. For any fixed $c>0$ let $\Delta_{n}(c)$ be defined by

$$
\Delta_{n}(c)=\left[-1+c n^{-2}, 1-c n^{-2}\right] \backslash \bigcup_{k \in \tau}\left[t_{k}-c n^{-1}, t_{k}+c n^{-1}\right] .
$$

$n=1,2, \ldots$ If $w \in G S J$ then also $\tilde{w} \in G S J$ where $\tilde{w}(x)=\left(1-x^{2}\right) w(x)$. If $1_{n}^{c}$ is the characteristic function of $\Delta_{n}(c)$ then by (15)

$$
\left|1_{n}^{c}(x) p_{n}(w, x)\right| \leqslant \text { const }\left[w(x) \sqrt{1-x^{2}}\right]^{-1 / 2}, \quad|x| \leqslant 1,
$$

and

$$
\left|1_{n}^{c}(x) p_{n}(\tilde{w}, x)\right| \leqslant \text { const }\left[w(x)\left(1-x^{2}\right)^{3 / 2}\right]^{-1 / 2}, \quad|x| \leqslant 1 .
$$

Our first goal is to prove that for every fixed $c>0$

$$
\sup _{n \geqslant 1} \| L_{n}(w, \text { of }) 1_{n}^{c} u \|_{p} \leqslant \text { const }\|f\|_{\infty} \quad \text { for } 1<p<\infty .
$$

In the following we will denote by $G_{i}(i=1,2, \ldots, 5)$ functions which might depend on $n$ and which have the properties that $\left|G_{i}(x)\right| \leqslant 1$ almost everywhere in $[-1,1]$ and $G_{i}(x)=0$ for $|x|>1$. Then

$$
\int\left|L_{n}(w, v f)\right|^{p} u^{p} 1_{n}^{c}=\int L_{n}(w, v f) G_{1}\left|L_{n}(w, v f)\right|^{p-1} u^{p} 1_{n}^{c}
$$

so that by (3)

$$
\begin{gathered}
\int\left|L_{n}(w, v f)\right|^{p} u^{p} 1_{n}^{c}=\frac{\gamma_{n-1}}{\gamma_{n}} \sum_{k=1}^{n} \lambda_{k n} p_{n-1}\left(w, x_{k n}\right) v\left(x_{k n}\right) f\left(x_{k n}\right) \\
\cdot \int_{-1}^{1} \frac{p_{n}(w, x)}{x-x_{k n}} G_{1}(x)\left|L_{n}(w, v f, x)\right|^{p-1} u(x)^{p} 1_{n}^{c}(x) d x
\end{gathered}
$$


where $\gamma_{n}=\gamma_{n}(w), \lambda_{k n}(w)$ and $x_{k n}=x_{k n}(w)$. Let $\rho_{n}$ be an arbitrary polynomial of degree at most $n$ which is positive on $\Delta_{n}(c)$. If $\Pi_{2 n}$ is defined by

$$
\begin{aligned}
\Pi_{2 n}= & \rho_{n} p_{n}(w) H\left(G_{1} \mid\left. L_{n}(w, \text { vf })\right|^{p-1} u^{p} 1_{n}^{c} \rho_{n}^{-1}\right) \\
& -H\left(p_{n}(w) G_{1}\left|L_{n}(w, v f)\right|^{p-1} u^{p} 1_{n}^{c}\right)
\end{aligned}
$$

then

$$
\begin{aligned}
\Pi_{2 n}(t)= & \int_{-1}^{1} \frac{\rho_{n}(x) p_{n}(w, x)-\rho_{n}(t) p_{n}(w, t)}{x-t} \\
& \cdot G_{1}(x)\left|L_{n}(w, v f, x)\right|^{p-1} u(x)^{p} \rho_{n}(x)^{-1} 1_{n}^{c}(x) d x
\end{aligned}
$$

so that $\Pi_{2 n}$ is a polynomial of degree less than $2 n$ and

$$
\Pi_{2 n}\left(x_{k n}\right)=\int_{-1}^{1} \frac{p_{n}(w, x)}{x-x_{k n}} G_{1}(x)\left|L_{n}(w, v f, x)\right|^{p-1} u(x)^{p} 1_{n}^{c}(x) d x .
$$

Thus we can rewrite (52) as

$$
\int\left|L_{n}(w, v f)\right|^{p} u^{p} 1_{n}^{c}=\frac{\gamma_{n-1}}{\gamma_{n}} \sum_{k=1}^{n} \lambda_{k n} p_{n-1}\left(w, x_{k n}\right) v\left(x_{k n}\right) f\left(x_{k n}\right) \Pi_{2 n}\left(x_{k n}\right) .
$$

Applying (1) and (19) we obtain

$$
\int\left|L_{n}(w, v f)\right|^{p} u^{p} 1_{n}^{c} \leqslant \mathrm{const}\|f\|_{\infty} \sum_{k=1}^{n} \lambda_{k n}\left|p_{n-1}\left(\tilde{w}, x_{k n}\right)\right|\left(1-x_{k n}^{2}\right) v\left(x_{k n}\right)\left|\Pi_{2 n}\left(x_{k n}\right)\right|
$$

and by (24)

$$
\int\left|L_{n}(w, v f)\right|^{p} u^{p} 1_{n}^{c} \leqslant \mathrm{const}\|f\|_{\infty} \int_{-1}^{1}\left|p_{n-1}(\tilde{w}) \Pi_{2 n}\right| v \tilde{w}
$$

Since $v \sqrt{w \sqrt{1-x^{2}}}$ is integrable, so is $v \tilde{w}$. Hence by (25) there exists a number $\bar{c}>0$ such that if $1_{n}^{\bar{c}}=1_{\Delta_{n}(\bar{c})}$ then

$$
\int\left|L_{n}(w, v f)\right|^{p} u^{p} 1_{n}^{c} \leqslant \text { const }\|f\|_{\infty} \int\left|p_{n-1}(\tilde{w}) \Pi_{2 n}\right| v \tilde{w} 1_{n}^{\bar{c}}
$$

Using the definition (53) of $\Pi_{2 n}$ we obtain

$$
\begin{aligned}
& \int\left|L_{n}(w, v f)\right|^{p} u^{p} 1_{n}^{c} \\
& \leqslant \text { const }\|f\|_{\infty}\left[\int\left|p_{n-1}(\tilde{w}) p_{n}(w) \rho_{n} v \tilde{w} 1_{n}^{\bar{c}} H\left(G_{1}\left|L_{n}(w, v f)\right|^{p-1} u^{p} 1_{n} \rho_{n}^{-1}\right)\right|\right. \\
& \left.+\int\left|p_{n-1}(\tilde{w}) v \tilde{w} 1_{n}^{\bar{c}} H\left(p_{n}(w) G_{1}\left|L_{n}(w, v f)\right|^{p-1} u^{p} 1_{n}^{c}\right)\right|\right] \\
& =\text { const }\|f\|_{\infty}\left[I_{1}+I_{2}\right] \text {. }
\end{aligned}
$$


Let us choose the polynomial $\rho_{n}$ as follows. First pick a positive integer $M$ such that $\left(1-x^{2}\right)^{M-1 / 2} v(x)=\tilde{v}(x)$ is continuous in $[-1,1]$. Let $\bar{n}=[n / 2-M]$ and let $\rho_{n}$ be defined by

$$
\rho_{n}(x)=(\bar{n})^{-1}\left(1-x^{2}\right)^{M} \lambda_{\bar{n}}(\tilde{v}, x)^{-1} .
$$

Then $\rho_{n}$ is a polynomial of degree $2 M+2(\bar{n}-1) \leqslant n$ and by (13)

$$
\rho_{n}(x) \leqslant \text { const } v(x)^{-1}, \quad|x| \leqslant 1,
$$

and

$$
\rho_{n}(x)^{-1} \leqslant \text { const } v(x), \quad|x| \leqslant 1-c n^{-2},
$$

for every fixed $c>0$. Now we are ready to estimate $I_{1}$ in (54). Applying (49), (50), (55) and (56) we get

$$
\begin{aligned}
I_{1} & \leqslant \text { const } \int_{-1}^{1}\left|H\left(G_{2}\left|L_{n}(w, v f)\right|^{p-1} u^{p} v 1_{n}^{c}\right)\right| \\
& =\text { const } \int_{-1}^{1} G_{3} H\left(G_{2}\left|L_{n}(w, v f)\right|^{p-1} u^{p} v 1_{n}^{c}\right) \\
& =-\operatorname{const} \int_{-1}^{1} H\left(G_{3}\right) G_{2}\left|L_{n}(w, v f)\right|^{p-1} u^{p} v 1_{n}^{c} \\
& \leqslant \text { const } \int_{-1}^{1}\left|H\left(G_{3}\right) L_{n}(w, v f)^{p-1} v u^{p} 1_{n}^{c}\right|
\end{aligned}
$$

Thus by Hölder's inequality

$$
I_{1} \leqslant \text { const }\left\|L_{n}(w, v f) 1_{n}^{c} u\right\|_{p}^{p-1}\left\|H\left(G_{3}\right) v u\right\|_{p} .
$$

Now we can apply the Lemma with $\phi \equiv 1$ and $g=v u$ to conclude that

$$
I_{1} \leqslant \text { const }\left\|L_{n}(w, v f) 1_{n}^{c} u\right\|_{p}^{p-1} \cdot\left[1+\left\|v u\left(1+\log ^{+} v u\right)\right\|_{p}\right]
$$

so that

$$
I_{1} \leqslant \text { const } \| L_{n}(w, \text { vf }) 1_{n}^{c} u \|_{p}^{p-1} .
$$

Estimating $I_{2}$ in (54) goes along similar lines. We have by (49) and (50)

$$
I_{2} \leqslant \text { const } \int v w_{*}\left|H\left(w_{*}^{-1} G_{4}\left|L_{n}(w, v f)\right|^{p-1} u^{p} 1_{n}^{c}\right)\right|
$$

where $w_{*}$ is defined by $w_{*}(x)=\sqrt{w(x) \sqrt{1-x^{2}}}$. Thus

$$
\begin{aligned}
I_{2} & \leqslant \text { const } \int_{-1}^{1} v w_{*} G_{5} H\left(w_{*}^{-1} G_{4}\left|L_{n}(w, v f)\right|^{p-1} u^{p} 1_{n}^{c}\right) \\
& =-\operatorname{const} \int_{-1}^{1} H\left(v w_{*} G_{5}\right) w_{*}^{-1} G_{4}\left|L_{n}(w, v f)\right|^{p-1} u^{p} 1_{n}^{c} \\
& \leqslant \text { const } \int_{-1}^{1}\left|H\left(v w_{*} G_{5}\right)\right| w_{*}^{-1}\left|L_{n}(w, v f)\right|^{p-1} u^{p} 1_{n}^{c}
\end{aligned}
$$

so that by Hölder's inequality

$$
I_{2} \leqslant \text { const }\left\|L_{n}(w, v f) 1_{n}^{c} u\right\|_{p}^{p^{-1}}\left\|v u\left(v w_{*}\right)^{-1} H\left(v w_{*} G_{5}\right)\right\|_{p} .
$$


By the conditions $v w_{*} \in L^{1}$ so that $v w_{*} \in G J$ and we can apply the Lemma with $\dot{\phi}=v w_{*}$ and $g=u v$ to obtain

$$
I_{2} \leqslant \text { const }\left\|L_{n}(w, v f) 1_{n}^{c} u\right\|_{p}^{p-1}\left[1+\left\|u v\left(1+v^{-1} w_{*}^{-1}+\log ^{+} u v\right)\right\|_{p}\right] .
$$

Consequently,

$$
I_{2} \leqslant \text { const } \| L_{n}(w, \text { vf }) 1_{n}^{c} u \|_{p}^{p-1} .
$$

Substituting (57) and (58) into (54), we see that inequality (51) holds. Our next step is to show that

$$
\sup _{n \geqslant 1}\left\|L_{n}(w, v f)\left(1-1_{n}^{c}\right) u\right\|_{p} \leqslant \text { const }\|f\|_{\infty}
$$

holds as well for $1<p<\infty$ and some $c>0$. Let us estimate the sum

$$
\sum_{k=1}^{n} \lambda_{k n}\left|p_{n-1}\left(w, x_{k n}\right)\right| v\left(x_{k n}\right)=S \text {. }
$$

If $\tilde{w}$, as earlier, is defined by $\tilde{w}(x)=\left(1-x^{2}\right) w(x)$ then by (19) and (24)

$$
S \leqslant \text { const } \int_{-1}^{1}\left|p_{n-1}(\tilde{w}, x)\right| v(x)\left(1-x^{2}\right) w(x) d x .
$$

Thus by (16)

$$
S \leqslant \text { const } \int_{-1}^{1}\left[1+\left(w(x)\left(1-x^{2}\right)^{3 / 2}\right)^{-1 / 2}\right] v(x)\left(1-x^{2}\right) w(x) d x
$$

and by the conditions of the theorem we get

$$
\sum_{k=1}^{n} \lambda_{k n}\left|p_{n-1}\left(w, x_{k n}\right)\right| v\left(x_{k n}\right) \leqslant \text { const. }
$$

The next sum to be estimated is

$$
\sum_{x_{k n} \geqslant \varepsilon} \lambda_{k n}\left|p_{n-1}\left(w, x_{k n}\right)\right| v\left(x_{k n}\right)\left(1-x_{k n}\right)^{-1}=T
$$

where $1>\varepsilon>0$ is chosen so that $t_{1}$ in (47) is less than $\varepsilon$. Since $v$ is a Jacobi weight, we have $v(x)=(1-x)^{a}(1+x)^{b}$ for some $a$ and $b$. Taking the choice of $\varepsilon$ into consideration, and using (11), (14) and (20) we obtain

$$
T \leqslant \text { const } \frac{1}{n} \sum_{x_{k n} \geqslant \varepsilon}\left(1-x_{k n}\right)^{\left(\Gamma_{0}+2 a-1 / 2\right) / 2}
$$

and since $1-x_{k n} \sim k^{2} / n^{2}$ by (11), we get

$$
T \leqslant \text { const } n^{-\Gamma_{0}-2 a-1 / 2} \sum_{k=1}^{n} k^{\Gamma_{0}+2 a-1 / 2} .
$$

Hence

$$
T \leqslant \text { const } \begin{cases}1, & \Gamma_{0}+2 a-1 / 2>-1 \\ \log (n+1), & \Gamma_{0}+2 a-1 / 2=-1 \\ n^{\Gamma_{0}-2 a-1 / 2}, & \Gamma_{0}+2 a-1 / 2<-1\end{cases}
$$


Now we turn to estimating $L_{n}(w, v f, x)$ for $1+x_{1 n} \leqslant 2 x \leqslant 2$. By (1) and (3) we have

$$
\begin{aligned}
L_{n}(w, v f, x) \mid \leqslant 2\|f\|_{\infty} p_{n}(x)[ & \frac{1}{\varepsilon} \sum_{k=1}^{n} \lambda_{k n}\left|p_{n-1}\left(w, x_{k n}\right)\right| v\left(x_{k n}\right) \\
& \left.+\sum_{x_{k n} \geqslant \varepsilon} \lambda_{k n}\left|p_{n-1}\left(w, x_{k n}\right)\right| v\left(x_{k n}\right)\left(1-x_{k n}\right)^{-1}\right], \\
\quad & 1+x_{1 n} \leqslant 2 x \leqslant 2 .
\end{aligned}
$$

where $\varepsilon$ is chosen as in (61). Hence by (15) and (60)-(62)

$$
\left|L_{n}(w, v f, x)\right| \leqslant \mathrm{const}\|f\|_{\infty} \begin{cases}n^{\Gamma_{0}+1 / 2}, & \Gamma_{0}+2 a-1 / 2>-1, \\ n^{-2 a} \log (n+1), & \Gamma_{0}+2 a-1 / 2=-1, \\ n^{\Gamma_{0}+1 / 2}+n^{-2 a}, & \Gamma_{0}+2 a-1 / 2<-1,\end{cases}
$$

for $1+x_{1 n} \leqslant 2 x \leqslant 2$, and consequently

$$
\left|L_{n}(w, v f, x)\right| \leqslant \text { const }\|f\|_{\infty}\left[n^{\Gamma_{0}+1 / 2}+n^{-2 a} \log (n+1)\right], \quad 1+x_{1 n} \leqslant 2 x \leqslant 2 .
$$

If $a>0$ then from (63) we get

$$
\left|L_{n}(w, v f, x)\right| \leqslant \text { const }\|f\|_{\infty}\left[1+1 / \sqrt{w(x) \sqrt{1-x^{2}}}\right], \quad 1+x_{1 n} \leqslant 2 x \leqslant 2,
$$

and thus

$$
\begin{aligned}
& \int_{\left(1+x_{1 n}\right) / 2}^{1}\left|L_{n}(w, v f, x) u(x)\right|^{p} d x \\
& \leqslant \text { const }\|f\|_{\infty}^{p} \int_{\left(1+x_{1 n}\right) / 2}^{1}\left[1+\frac{1}{\sqrt{w(x) \sqrt{1-x^{2}}}}\right]^{p} u(x)^{p} d x .
\end{aligned}
$$

If $a \leqslant 0$ then by (11) and (63)

$$
\begin{array}{rl}
\left|L_{n}(w, v f, x)\right| \leqslant \text { const }\|f\|_{\infty}\left[1+1 / \sqrt{w(x) \sqrt{1-x^{2}}}\right] & +v(x) \log (n+1)], \\
1 & 1+x_{1 n} \leqslant 2 x \leqslant 2
\end{array}
$$

and consequently

$$
\begin{aligned}
& \left|L_{n}(w, v f, x) u(x)\right| \\
& \leqslant \text { const }\|f\|_{\infty}\left[u(x)+u(x) / \sqrt{w(x) \sqrt{1-x^{2}}}\right. \\
& \left.+2 p u(x) v(x) \log ^{+} u(x) v(x)+(n+1)^{1 / 2 p} \log (n+1)\right], \\
& 1+x_{1 n} \leqslant 2 x \leqslant 2 .
\end{aligned}
$$


Hence

(65)

$$
\begin{aligned}
& \int_{\left(1+x_{1 n}\right) / 2}^{1}\left|L_{n}(w, v f, x) u(x)\right|^{p \leqslant \text { const }\|f\|_{\infty}} \\
& \cdot\left[\int_{\left(1+x_{1 n}\right) / 2}^{1}\left[u(x)+\frac{u(x)}{\sqrt{w(x) \sqrt{1-x^{2}}}}+2 p u(x) v(x) \log ^{+} u(x) v(x)\right]^{p} d x\right. \\
& \left.+\left(1-x_{1 n}\right) 2^{-1}(n+1)^{1 / 2}(\log (n+1))^{p}\right] .
\end{aligned}
$$

Since by (11), $1-x_{1 n} \leqslant$ const $n^{-2}$, we obtain from (64) and (65) that

$$
\int_{\left(1+x_{1 n}\right) \mid 2}^{1}\left|L_{n}(w, v f) u\right|^{p} \leqslant \text { const }\|f\|_{\infty}^{p}
$$

whenever $w, v$ and $u$ satisfy the conditions of the theorem. The inequality

$$
\int_{-1}^{\left(-1+x_{n n}\right) / 2} \mid\left. L_{n}(w, \text { of }) u\right|^{p} \leqslant \mathrm{const}\|f\|_{\infty}^{p}
$$

can be proved by similar arguments. Now let $t_{j}$ be a singularity of $w$ in (47) such that $j \in \tau$ (see (48)), in another words let $t_{j}$ be such that the corresponding $\Gamma_{j}$ is negative. We will show that

$$
\left|L_{n}(w, v f, x)\right| \leqslant \text { const }|| f \|_{\infty}, \quad\left|x-t_{j}\right| \leqslant 1 / n .
$$

Let $\delta>0$ be chosen so that $t_{j+1}<t_{j}-2 \delta<t_{j}+2 \delta<t_{j-1}$ with the notation $t_{m+1}=-1$ and $t_{0}=1$. Then by (11) there exist a uniformly bounded number of roots $x_{k n}$ such that $\left|x-x_{k n}\right| \leqslant 2 / n$ if $\left|x-t_{j}\right| \leqslant 1 / n$. Hence

$$
\begin{aligned}
\sum_{\left|x-x_{k n}\right| \leqslant 2 / n}\left|l_{k n}(x)\right| & \leqslant\left[\sum_{\left|x-x_{k n}\right| \leqslant 2 / n} \lambda_{k n} \sum_{k=1}^{n} \frac{l_{k n}(x)^{2}}{\lambda_{k n}}\right]^{1 / 2} \\
& =\left[\sum_{\left|x-x_{k n}\right| \leqslant 2 / n} \lambda_{k n} \cdot \lambda_{n}(x)^{-1}\right]^{1 / 2}, \quad\left|x-t_{j}\right| \leqslant 1 / n,
\end{aligned}
$$

and by (13)

$$
\sum_{\left|x-x_{k n}\right| \leqslant 2 / n}\left|l_{k n}(x)\right| \leqslant \text { const, } \quad\left|x-t_{j}\right| \leqslant 1 / n .
$$

If $\left|x-t_{j}\right| \leqslant 1 / n$ and $\left|x-x_{k n}\right|>2 / n$ then $\left|t_{j}-x_{k n}\right| \leqslant 3\left|x-x_{k n}\right| / 2$. Thus by (1), (3), (14) and (15)

$$
\sum_{2 / n \leqslant\left|x-x_{k n}\right| \leqslant \delta}\left|l_{k n}(x)\right| \leqslant \text { const } n^{\Gamma_{j} / 2-1} \sum_{2 / n<\left|x-x_{k n}\right| \leqslant \delta}\left|t_{j}-x_{k n}\right|^{\Gamma, / 2-1} .
$$


We can estimate $\left|t_{j}-x_{k n}\right|$ by (11) we obtain

$$
\sum_{2 / n \leqslant\left|x-x_{k n}\right| \leqslant \delta}\left|l_{k n}(x)\right| \leqslant \operatorname{const} n^{\Gamma_{j} / 2-1} \sum_{l=1}^{n}\left(\frac{l}{n}\right)^{\Gamma_{,} / 2-1} .
$$

Since $\Gamma_{j}<0$, we can conclude that

$$
\sum_{2 / n \leqslant\left|x-x_{k n}\right| \leqslant \delta}\left|l_{k n}(x)\right| \leqslant \text { const, } \quad\left|x-t_{j}\right| \leqslant \frac{1}{n} .
$$

Applying (1), (15) and (60) we see that

$$
\sum_{\delta<\left|x-x_{k n}\right|} v\left(x_{k n}\right)\left|l_{k n}(x)\right| \leqslant \operatorname{const} n^{\Gamma_{j} / 2}, \quad\left|x-t_{j}\right| \leqslant \frac{1}{n} .
$$

By the choice of $\delta$ the function $v$ is uniformly bounded on $\left[t_{j}-2 \delta, t_{j}+2 \delta\right] \supset[x-$ $\delta, x+\delta]$ if $\left|x-t_{j}\right| \leqslant 1 / n$. Because $\Gamma_{j}<0$, we obtain from (69)-(71) that (68) holds, and consequently

$$
\int_{t_{j}-1 / n}^{t_{j}+1 / n}\left|L_{n}(w, v f) u\right|^{p} \leqslant \text { const }\|f\|_{\infty}^{p}
$$

Now we choose $1>c>0$ so that $\left(-1+x_{n n}\right) / 2>-1+c n^{-2},\left(1+x_{1 n}\right) / 2<1-$ $c n^{-2}$. The existence of such a number $c$ is guaranteed by (11). Since $\tau$ in (48) contains no more than $m$ indices, inequality (59) follows from (66), (67) and (72). For $1<p<\infty$ the theorem follows from (51) and (59). Now let $0<p \leqslant 1$. Define $\bar{u}$ by

$$
\bar{u}=u^{p / 2}\left[1+\left(\sqrt{w \sqrt{1-x^{2}}}\right)^{(p-2) / 2}+v^{(2-p) / 2}\left(1+\left(\log ^{+} v u\right)^{(2-p) / 2}\right)\right]^{-1} .
$$

Then $\bar{u} \leqslant u^{p / 2}$ and $\bar{u} \leqslant u^{p / 2}\left(\sqrt{w \sqrt{1-x^{2}}}\right)^{(2-p) / 2}$ so that $\bar{u} \in L^{2}$ and $\left(\sqrt{w \sqrt{1-x^{2}}}\right)^{-1} \bar{u} \in L^{2}$. Moreover, $v \bar{u} \leqslant(v u)^{p / 2}$ and $v \bar{u} \leqslant(v u)^{p / 2}\left(\log ^{+} v u\right)^{(p-2) / 2}$. Thus $v \bar{u} \log ^{+} v \bar{u} \leqslant(p / 2)(v u)^{p / 2}\left(\log ^{+} v u\right)^{p / 2}$ so that $v \bar{u} \in L^{2}\left(\log ^{+} L\right)^{2}$. Hence we can apply (46) with $p=2$ to obtain

$$
\left\|L_{n}(v f) \bar{u}\right\|_{2} \leqslant \text { const }\|f\|_{\infty} .
$$

Since

$$
\left(u \bar{u}^{-1}\right)^{2 p /(2-p)} \leqslant 4^{2 p /(2-p)} u^{p}\left[1+\left(\sqrt{w \sqrt{1-x^{2}}}\right)^{-p}+v^{p}+v^{p}\left(\log ^{+} v u\right)^{p}\right],
$$

we have $u \bar{u}^{-1} \in L^{2 p(2-p)}$. By Hölder's inequality

$$
\left\|L_{n}(v f) u\right\|_{p} \leqslant\left\|L_{n}(v f) \bar{u}\right\|_{2}\left\|u \bar{u}^{-1}\right\|_{2 p /(2-p)},
$$

and consequently, the theorem also follows for $0<p \leqslant 1$.

THEOREM 2. Let $w \in G S J$ and $0<p<\infty$. Let $v$ be a not necessarily integrable Jacobi weight function and let $u$ be a measurable nonnegative function in $[-1,1]$ such that $u$ is positive on a set with positive measure. Suppose that

$$
\sup _{n \geqslant 1} \| L_{n}(w, \text { of }) u \|_{p} \leqslant \text { const }\|f\|_{\infty}
$$


for every continuous function f vanishing at \pm 1 . Then $u \in L^{p}, u v \in L^{p}$ and

$$
v \sqrt{w \sqrt{1-x^{2}}} \in L^{1} \text { and } u / \sqrt{w \sqrt{1-x^{2}}} \in L^{p} \text {. }
$$

Moreover, there exists a nonnegative $u$ such that $u \in L^{p}, u v \in L^{p} \backslash\left(L \log ^{+} L\right)^{p}$ and if (74) holds then (73) is not necessarily true for every continuous function vanishing at $\pm 1$

Proof. It is obvious that $u \in L^{p}$ whenever (73) holds. In order to show that $u v \in L^{p}$ as well as notice that if $f v$ is continuous then by Erdös-Turán's $L^{2}$ theorem $[6$, p. 146]

$$
\left.\lim _{n \rightarrow \infty} \| L_{n}(w, v f)-v f\right] w \|_{2}=0
$$

so that there exists a subsequence $\left\{n_{k}\right\}$ such that

$$
\lim _{k \rightarrow \infty} L_{n_{k}}(w, v f, x)=v(x) f(x)
$$

for almost every $x$ in $[-1,1]$. By (73) $\sup _{k \geqslant 1}\left\|L_{n_{k}}(w, v f) u\right\|_{p} \leqslant$ const $\|f\|_{\infty}$ for such a function $f$ if $f( \pm 1)=0$ and by Fatou's lemma

$$
\|v f u\|_{p} \leqslant \text { const }\|f\|_{\infty}
$$

whenever $f v$ is continuous and $f$ vanishes at \pm 1 . Now we choose a sequence $\left\{f_{j}\right\}$ such that $f_{j}( \pm 1)=0,\left\|f_{j}\right\|_{\infty} \leqslant 1, f_{j} v \in C[-1,1]$ and $f_{j}(x) \rightarrow_{j \rightarrow \infty} 1$ for every $x \in$ $(-1,1)$. Then by (75) $\sup _{j \geqslant 1}\left\|f_{j} v u\right\|_{p} \leqslant$ const and another application of Fatou's lemma yields $u v \in L^{p}$. Now suppose that (73) holds but $v \sqrt{w \sqrt{1-x^{2}}}$ is not integrable. If $w$ is given by (10) and $v$ is defined by $v(x)=(1-x)^{a}(1+x)^{b}$, then this amounts to either $a+\Gamma_{0} / 2+5 / 4<0$ or $b+\Gamma_{m+1} / 2+5 / 4<0$. Let $0<\varepsilon<1$ be chosen so that $t_{1}<\varepsilon$ and $-\varepsilon<t_{m}$ where $t_{1}$ and $t_{m}$ are given in (10). Then by (11), (14) and (20)

$$
\begin{aligned}
\sum_{\left|x_{k n}\right| \geqslant \varepsilon} \lambda_{k n} \mid p_{n-1} & \left(w, x_{k n}\right) \mid v\left(x_{k n}\right) \\
& \sim \frac{1}{n} \sum_{x_{k n} \leqslant-\varepsilon}\left(1+x_{k n}\right)^{b+\Gamma_{m+1} / 2+3 / 4}+\frac{1}{n} \sum_{x_{k n} \geqslant \varepsilon}\left(1-x_{k n}\right)^{a+\Gamma_{0} / 2+3 / 4} \\
& \sim \frac{1}{n} \sum_{x_{k n} \leqslant-\varepsilon}\left(\frac{k}{n}\right)^{2 b+\Gamma_{m+1}+3 / 2}+\frac{1}{n} \sum_{x_{k n} \geqslant \varepsilon}\left(\frac{k}{n}\right)^{2 a+\Gamma_{0}+3 / 2}
\end{aligned}
$$

and since either $2 b+\Gamma_{m+1}+3 / 2<-1$ or $2 a+\Gamma_{0}+3 / 2<-1$, we have

$$
\lim _{n \rightarrow \infty} \sum_{\left|x_{k n}\right| \geqslant \varepsilon} \lambda_{k n}\left|p_{n-1}\left(w, x_{k n}\right)\right| v\left(x_{k n}\right)=\infty
$$

Let $\left\{f_{n}\right\}$ be a sequence of continuous functions such that $f_{n}( \pm 1)=0, f_{n}\left(x_{k n}\right)=0$ for $\left|x_{k n}\right|<\varepsilon, f_{n}\left(x_{k n}\right)=-\operatorname{sign} p_{n-1}\left(w, x_{k n}\right)$ for $\varepsilon \leqslant x_{k n}<1, f_{n}\left(x_{k n}\right)=\operatorname{sign} p_{n-1}\left(w, x_{k n}\right)$ 
for $-1<x_{k n} \leqslant-\varepsilon$ and $\left|f_{n}(x)\right| \leqslant 1$ for every $x$ in $[-1,1]$. Using (3), we have

$$
\begin{aligned}
L_{n}\left(w, v f_{n}, x\right)=\frac{\gamma_{n-1}}{\gamma_{n}} p_{n}(w, x) & {\left[\sum_{x_{k n} \geqslant \varepsilon} \lambda_{k n}\left|p_{n-1}\left(w, x_{k n}\right)\right| v\left(x_{k n}\right)\left(x_{k n}-x\right)^{-1}\right.} \\
& \left.+\sum_{x_{k n} \leqslant-\varepsilon} \lambda_{k n}\left|p_{n-1}\left(w, x_{k n}\right)\right| v\left(x_{k n}\right)\left(x-x_{k n}\right)^{-1}\right]
\end{aligned}
$$

and consequently

$$
\left|L_{n}\left(w, v f_{n}, x\right)\right| \geqslant \frac{1}{2} \frac{\gamma_{n-1}}{\gamma_{n}}\left|p_{n}(w, x)\right| \sum_{\left|x_{k n}\right| \geqslant \varepsilon} \lambda_{k n}\left|p_{n-1}\left(w, x_{k n}\right)\right| v\left(x_{k n}\right)
$$

for $|x| \leqslant \varepsilon$. Applying (21), (73), (76) and (77) we obtain

$$
\lim _{n \rightarrow \infty} \int_{-\varepsilon}^{\varepsilon} \mid p_{n}(w, x) u(x) p d x=0
$$

and by [13, Theorem 4.2.8, p. 42] $u(x)=0$ for almost every $x$ in $[-\varepsilon, \varepsilon]$. Letting $\varepsilon \rightarrow 1$ we see that $u$ must vanish almost everywhere in $[-1,1]$ whenever $v \sqrt{w \sqrt{1-x^{2}}}$ is not integrable, and this contradicts to the conditions of the theorem. Now assume that (73) holds whereas $u / \sqrt{w \sqrt{1-x^{2}}}$ does not belong to $L^{p}$. Then $[13$, Theorem 7.32, p. 138]

$$
\lim _{n \rightarrow \infty}\left\|p_{n}(w) u\right\|_{p}=\infty .
$$

Hence we can choose an interval $\Delta$ such that either $\Delta=[-1,0]$ or $\Delta=[0,1]$ and

$$
\lim _{n \rightarrow \infty} \sup \left\|p_{n}(w) u 1_{\Delta}\right\|=\infty
$$

where $1_{\Delta}$ is the characteristic function of $\Delta$. Let $\left\{f_{n}\right\}$ be a sequence of continuous functions such that $f_{n}( \pm 1)=0, f_{n}\left(x_{k n}\right)=\operatorname{sign} p_{n-1}\left(w, x_{k n}\right) \cdot\left[1-1_{\Delta}\left(x_{k n}\right)\right]$ and $\left|f_{n}(x)\right| \leqslant 1$ for $-1 \leqslant x \leqslant 1$. Then by (3)

$$
L_{n}\left(w, v f_{n}, x\right)=\frac{\gamma_{n-1}}{\gamma_{n}} p_{n}(w, x) \sum_{x_{k n} \notin \Delta} \lambda_{k n}\left|p_{n-1}\left(w, x_{k n}\right)\right| v\left(x_{k n}\right)\left(x-x_{k n}\right)^{-1}
$$

and therefore

$$
\left|L_{n}\left(w, v f_{n}, x\right)\right| \geqslant \frac{1}{2} \frac{\gamma_{n-1}}{\gamma_{n}}\left|p_{n}(w, x)\right| \sum_{x_{k n} \notin \Delta} \lambda_{k n}\left|p_{n-1}\left(w, x_{k n}\right)\right| v\left(x_{k n}\right)
$$

for $x \in \Delta$. Now we can apply (21), (73) and (78) to obtain

$$
\liminf _{n \rightarrow \infty} \sum_{x_{k n} \notin \Delta} \lambda_{k n}\left|p_{n-1}\left(w, x_{k n}\right)\right| v\left(x_{k n}\right)=0 .
$$

Choose an interval $\Delta^{*}$ such that $\Delta^{*} \cap \Delta=0, \pm 1 \notin \Delta^{*}$ and if $w$ is defined by (10) then none of the singularities $t_{j}$ of $w$ belong to $\Delta^{*}$. Then

$$
\liminf _{n \rightarrow \infty} \sum_{x_{k n} \in \Delta^{*}} \lambda_{k n}\left|p_{n-1}\left(w, x_{k n}\right)\right| v\left(x_{k n}\right)=0 .
$$

and by (20)| $p_{n-1}\left(w, x_{k n}\right) \mid v\left(x_{k n}\right) \geqslant$ const for $x_{k n} \in \Delta^{*}$ so that

$$
\liminf _{n \rightarrow \infty} \sum_{x_{k n} \in \Delta^{*}} \lambda_{k n}=0 \text {. }
$$


By the quadrature convergence theorem [7, p. 89]

$$
\lim _{n \rightarrow x} \sum_{x_{k n} \in \Delta^{*}} \lambda_{k n}=\int_{\Delta^{*}} w
$$

and consequently

$$
\int_{\lrcorner^{*}} w=0
$$

so that $u(x)=0$ for almost every $x \in \Delta^{*}$. This contradiction shows that $u / \sqrt{u \sqrt{1-x^{2}}}$ must belong to $L^{p}$ whenever (73) is satisfied. In order to prove the last part of the theorem we assume that if $u \in L^{p}, u v \in L^{p}$ and (74) is satisfied then (73) holds. First we fix an interval, say $\Delta$, such that $\pm 1 \notin \Delta$ and if $w$ is defined by (10) then none of the singularities $t_{j}$ of $w$ belong to $\Delta$. We will show that if $f$ is continuous in $[-1,1]$ and $f$ is supported in $\Delta$ then

$$
\lim _{n \rightarrow \infty}\left\|\left[L_{n}(w, v f)-v f\right] u\right\|_{p}=0
$$

if (73) holds. Since of is continuous in $[-1,1]$ and vanishes outside $\Delta$, for every $\delta>0$ we can pick a polynomial $R$ such that $v( \pm 1)^{-1} R( \pm 1)=0$ and $|v(x) f(x)-R(x)| \leqslant$ $\delta v(x)$ for $-1<x<1$. Hence by (73)

$$
\left\|\left[L_{n}(x, v f)-v f\right] u\right\|_{p} \leqslant \operatorname{const}\left\{\left\|\left[L_{n}(w, v f-R)\right] u\right\|_{p}+\|[v f-R] u\|_{p}\right\} \leqslant \text { const } \delta
$$

if $n>\operatorname{deg} R$. Therefore (79) is indeed true for continuous functions $f$ supported in $\Delta$ whenever (73) holds. Now let $\omega$ be an arbitrary $L^{1}$ function supported in $\Delta$. By the choice of $\Delta$, if $u=|\omega|^{1 / p}$ then $u \in L^{p}, u v \in L^{p}$ and $u / \sqrt{w \sqrt{1-x^{2}}} \in L^{p}$ so that by the assumptions made

$$
\lim _{n \rightarrow \infty} \int_{\Delta}\left|L_{n}(w, v f)-v f\right|^{p} \omega=0
$$

for every continuous function $f$ supported in $\Delta$. Thus by the Banach-Steinhaus theorem there exists a constant $K_{f}$ depending on $f$ but independent of $\omega$ such that

$$
\sup _{n \geqslant 1}\left|\int_{\Delta}\right| L_{n}(w, v f)-\left.v f\right|^{p} \omega\left|\leqslant K_{f} \int_{\Delta}\right| \omega \mid
$$

and taking the supremum of the left side over every $\omega$ with $\int_{\Delta}|\omega|=1$ we obtain

$$
\sup _{n \geq 1}\left\|L_{n}(w, v f)-v f\right\|_{\infty}^{p} \leqslant K_{f}
$$

where the $\infty$-norm is taken over $\Delta$, and $f$ is an arbitrary continuous function supported in $\Delta$. Let $C_{0}(\Delta)$ be the Banach space of all continuous functions supported on $\Delta$ such that the norm is defined by the maximum norm. For every $F \in C_{0}(\Delta)$ there exists a function $f$ such that $F=v f$ and $f \in C_{0}(\Delta)$. Hence (80) becomes

$$
\sup _{n \geqslant 1}\left\|L_{n}(w, F)-F\right\|_{\infty}<\infty
$$


for every $F \in C_{0}(\Delta)$ where the $\infty$-norm is taken over $\Delta$. Thus by the uniform boundedness theorem $[9$, p. 26]

$$
\sup _{n \geqslant 1} \max _{x \in \Delta}\left|L_{n}(w, F, x)\right| \leqslant \text { const } \max _{x \in \Delta}|F(x)|
$$

for every $F \in C_{0}(\Delta)$. Let $\Delta_{1} \subset \Delta$ and $\Delta_{2} \subset \Delta_{1}$ be proper closed subintervals of $\Delta$ and $\Delta_{1}$, respectively. Let $x \in \Delta_{2}$ be a root of $p_{n+1}(w)$ and let $j$ be the index of that zero of $p_{n}(w)$ which is closest to $x$. Then by (11)

$$
\left|x-x_{k n}\right| \leqslant \mathrm{const}|k-j| / n, \quad x_{k n} \in \Delta_{1}, k \neq j .
$$

Let $F \in C_{0}(\Delta)$ be chosen so that $F\left(x_{j n}\right)=0, F\left(x_{k n}\right)=0$ for $x_{k n} \in \Delta \backslash \Delta_{1}, F\left(x_{k n}\right)=$ $\operatorname{sign}\left[p_{n-1}\left(w, x_{k n}\right)\left(x-x_{k n}\right)\right]$ for $x_{k n} \in \Delta_{1}$ and $|F(t)| \leqslant 1$ for $t \in \Delta$. Then by (3)

$$
L_{n}(w, F, x)=\frac{\gamma_{n-1}}{\gamma_{n}} p_{n}(w, x) \sum_{\substack{x_{k n} \in \Delta_{1} \\ k \neq j}} \lambda_{k n}\left|p_{n-1}\left(w, x_{k n}\right)\left(x-x_{k n}\right)^{-1}\right|
$$

and by (14), (20), (21) and (82)

$$
\left|L_{n}(w, F, x)\right| \geqslant \text { const } \sum_{\substack{x_{k n} \in \Delta_{1} \\ k \neq j}}|k-j|^{-1} .
$$

Since by (11) the number of zeros $x_{k n}$ in $\Delta_{1}$ is greater than const $\cdot n$, we obtain

$$
\left|L_{n}(w, F, x)\right| \geqslant \text { const } \cdot \log n .
$$

Substituting (83) into (81) we get $\log n \leqslant$ const and since $n$ can be as large as we wish, we see that (73) cannot be true for every $u$ satisfying $u \in L^{p}, u v \in L^{p}$ and (74). The proof of the theorem has been completed.

THEOREM 3. Let $w, v, u$ and p satisfy the conditions of Theorem 1. Suppose that

$$
\lim _{n \rightarrow \infty}\left\|\left[L_{n}(w, v)-v\right] u\right\|_{p}=0 .
$$

Then for every continuous function $f$ in $[-1,1]$ we also have

$$
\lim _{n \rightarrow \infty}\left\|\left[L_{n}(w, v f)-v f\right] u\right\|_{p}=0 .
$$

Proof. By Theorem 1 we have to show that (85) holds when $f$ is a polynomial. By linearity we can assume that $f$ is of the form $f(x)=x^{l}$ where $l$ is a nonnegative integer. Since we assume that (84) holds, we only have to prove (85) for $l \geqslant 1$. If $f(x)=x^{l}, l \geqslant 1$, then

$$
L_{n}(w, v f)-v f=L_{n}(w, v f)-f L_{n}(w, v)+f\left[L_{n}(w, v)-v\right]
$$

and by (84) formula (85) holds if

$$
\lim _{n \rightarrow \infty}\left\|\left[L_{n}(w, v f)-f L_{n}(w, v)\right] u\right\|_{p}=0 .
$$

Hence our goal is to prove (86). We have

$$
\begin{aligned}
L_{n}(w, v f, x)-f(x) L_{n}(w, v, x) & =\sum_{k=1}^{n}\left[x_{k n}^{l}-x^{l}\right] v\left(x_{k n}\right) l_{k n}(w, x) \\
& =-\sum_{j=0}^{l-1} x^{l-1-j} \sum_{k=1}^{n}\left(x-x_{k n}\right) x_{k n}^{j} v\left(x_{k n}\right) l_{k n}(w, x) .
\end{aligned}
$$


Applying (3) we get

(87)

$$
\sum_{k=1}^{n}\left(x-x_{k n}\right) x_{k n}^{j} v\left(x_{k n}\right) l_{k n}(w, x)=\frac{\gamma_{n-1}}{\gamma_{n}} p_{n}(w, x) \sum_{k=1}^{n} x_{k n}^{j} v\left(x_{k n}\right) p_{n-1}\left(w, x_{k n}\right) \lambda_{k n} \text {. }
$$

Thus by (1), (16) and (87)

$$
\begin{aligned}
\left.\| L_{n}(w, v f)-f L_{n}(w, v)\right] u \|_{p} \leqslant \text { const } \cdot\left\|\left[1+\frac{1}{\sqrt{w \sqrt{1-x^{2}}}}\right] u\right\|_{p} \\
\cdot \max _{0 \leqslant j \leqslant l-1}\left|\sum_{k=1}^{n} x_{k n}^{j} v\left(x_{k n}\right) p_{n-1}\left(w, x_{k n}\right) \lambda_{k n}\right|
\end{aligned}
$$

so that (86) holds if

$$
\lim _{n \rightarrow \infty} \sum_{k=1}^{n} x_{k n}^{j} v\left(x_{k n}\right) p_{n-1}\left(w, x_{k n}\right) \lambda_{k n}=0, \quad j=0,1, \ldots
$$

Let $0<\varepsilon<1$ be chosen so that all the singularities $t_{k}$ of $w$ in (10) belong to $[-\varepsilon, \varepsilon]$. If $R$ is an arbitrary polynomial and $n>\operatorname{deg} R+1$ then by the Gauss-Jacobi quadrature formula

$$
\sum_{k=1}^{n} R\left(x_{k n}\right) p_{n-1}\left(w, x_{k n}\right) \lambda_{k n}=0
$$

Let $1_{\varepsilon}$ be the characteristic function of $[-\varepsilon, \varepsilon]$. Then

$$
\begin{aligned}
& \sum_{k=1}^{n} 1_{\varepsilon}\left(x_{k n}\right) x_{k n}^{j} v\left(x_{k n}\right) p_{n-1}\left(w, x_{k n}\right) \lambda_{k n} \\
& =\sum_{k=1}^{n}\left[1_{\varepsilon}\left(x_{k n}\right) x_{k n}^{j} v\left(x_{k n}\right)-R\left(x_{k n}\right)\right] p_{n-1}\left(w, x_{k n}\right) \lambda_{k n} .
\end{aligned}
$$

Applying Cauchy's inequality we obtain

$$
\begin{aligned}
& \left|\sum_{k=1}^{n} 1_{\varepsilon}\left(x_{k n}\right) x_{k n}^{j} v\left(x_{k n}\right) p_{n-1}\left(w, x_{k n}\right) \lambda_{k n}\right|^{2} \\
& \leqslant \sum_{k=1}^{n} \lambda_{k n} \sum_{k=1}^{n}\left[1_{\varepsilon}\left(x_{k n}\right) x_{k n}^{j} v\left(x_{k n}\right)-R\left(x_{k n}\right)\right]^{2} p_{n-1}\left(w, x_{k n}\right)^{2} \lambda_{k n} .
\end{aligned}
$$

By the Gauss-Jacobi quadrature formula

$$
\sum_{k=1}^{n} \lambda_{k n}=\|w\|_{1}
$$

Since $\left[1_{\varepsilon} x^{j} v-R\right]^{2}$ is Riemann integrable, by [13, Theorem 4.2.3, p. 39] the second sum on the right side of (90) converges as $n \rightarrow \infty$, and evaluating its limit by the 
same theorem we obtain

$$
\begin{array}{r}
\limsup _{n \rightarrow \infty}\left|\sum_{k=1}^{n} 1_{\varepsilon}\left(x_{k n}\right) x_{k n}^{j} v\left(x_{k n}\right) p_{n-1}\left(w, x_{k n}\right) \lambda_{k n}\right|^{2} \\
\leqslant \frac{2}{\pi}\|w\|_{1} \int_{-1}^{1}\left[1_{\varepsilon}(t) t^{j} v(t)-R(t)\right]^{2} \sqrt{1-t^{2}} d t
\end{array}
$$

and taking the infimum on the right side here with respect to $R$ we get

$$
\lim _{n \rightarrow \infty} \sum_{k=1}^{n} 1_{\varepsilon}\left(x_{k n}\right) x_{k n}^{j} v\left(x_{k n}\right) p_{n-1}\left(w, x_{k n}\right) \lambda_{k n}=0, \quad j=0,1 \ldots \ldots
$$

We have

$$
\left|\sum_{\left|x_{k n}\right| \geqslant \varepsilon} x_{k n}^{j} v\left(x_{k n}\right) p_{n-1}\left(w, x_{k n}\right) \lambda_{k n}\right| \leqslant \sum_{\left|x_{k n}\right| \geqslant \varepsilon} v\left(x_{k n}\right)\left|p_{n}{ }_{1}\left(w, x_{k n}\right)\right| \lambda_{k n}
$$

and by (14) and (20)

(92)

$$
\left|\sum_{\left|x_{k n}\right| \geqslant \varepsilon} x_{k n}^{j} v\left(x_{k n}\right) p_{n-1}\left(w, x_{k n}\right) \lambda_{k n}\right| \leqslant \frac{\mathrm{const}}{n} \sum_{\left|x_{k n}\right| \geqslant \varepsilon} v\left(x_{k n}\right) \sqrt{w\left(x_{k n}\right)}\left(1-x_{k n}^{2}\right)^{3 / 4} .
$$

Since $v$ is a Jacobi weight, $w$ behaves like a Jacobi weight on $[-1,-\varepsilon] \cup[\varepsilon, 1]$ and $v \sqrt{w \sqrt{1-x^{2}}} \in L^{1}$, there exists a number $\delta>-1$ such that

$$
v\left(x_{k n}\right) \sqrt{w\left(x_{k n}\right)}\left(1-x_{k n}^{2}\right)^{3 / 4} \leqslant \operatorname{const}\left(1-x_{k n}^{2}\right)^{\delta / 2}
$$

and by (11)

(93) $v\left(x_{k n}\right) \sqrt{w\left(x_{k n}\right)}\left(1-x_{k n}^{2}\right)^{3 / 4} \leqslant$ const $\left\{\begin{array}{l}(k / n)^{\delta}, \quad x_{k n} \geqslant-\varepsilon, \\ ((n+1-k) / n)^{\delta}, \quad x_{k n} \leqslant-\varepsilon .\end{array}\right.$

Also by (11) if $x_{k n} \geqslant \varepsilon$ then $1 \leqslant k \leqslant$ const $\sqrt{1-\varepsilon^{2}} \cdot n$ and if $x_{k n} \leqslant-\varepsilon$ then $1 \leqslant n+$ $1-k \leqslant$ const $\sqrt{1-\varepsilon^{2}} \cdot n$. Thus by (92) and (93)

$$
\left|\sum_{\left|x_{k n}\right| \geqslant \varepsilon} x_{k n}^{j} v\left(x_{k n}\right) p_{n-1}\left(w, x_{k n}\right) \lambda_{k n}\right| \leqslant \operatorname{const}(\delta+1)^{-1}\left(1-\varepsilon^{2}\right)^{(\delta+1) / 2} .
$$

Combining (91) and (94) we obtain

$$
\limsup _{n \rightarrow \infty}\left|\sum_{k=1}^{n} x_{k n}^{j} v\left(x_{k n}\right) p_{n-1}\left(w, x_{k n}\right) \lambda_{k n}\right| \leqslant \operatorname{const}(\delta+1)^{-1}\left(1-\varepsilon^{2}\right)^{(\delta+1) / 2}
$$

and letting $\varepsilon \rightarrow 1$ formula (89) follows and so does (86).

THEOREM 4. Let $w \in G S J$ and $0<p<\infty$. Let $v$ be a not necessarily integrable rational Jacobi weight function, and let $u$ be a nonnegative function supported in $[-1,1]$ such that $u \in L^{p}, u v \in L^{p}, u / \sqrt{w \sqrt{1-x^{2}}} \in L^{p}$ and $v \sqrt{w \sqrt{1-x^{2}}} \in L^{1}$. Then

$$
\lim _{n \rightarrow \infty}\left\|\left[L_{n}(w, v)-v\right] u\right\|_{p}=0 \text {. }
$$


Proof. Let $v(x)=(1-x)^{a}(1+x)^{b}$ where $a$ and $b$ are integers. If $a \geqslant 0$ and $b \geqslant 0$ then $v$ is a polynomial and thus (95) holds. If $a<0$ and $b \geqslant 0$ then we proceed as follows. Choosing $n>b$ we see that

$$
(1-x)^{-a}\left[L_{n}(w, v, x)-v(x)\right]
$$

is a polynomial of degree $-a+n-1$ vanishing at the zeros of $p_{n}(w, x)$. Hence there exists a polynomial $R_{-a-1}$ of degree $-a-1$ such that

$$
(1-x)^{-a}\left[L_{n}(w, v, x)-v(x)\right]=p_{n}(w, x) R_{-a-1}(x) .
$$

The polynomial $R_{-a-1}$ can explictly be determined by dividing both sides by $p_{n}(w)$, expanding them in powers of $(x-1)$ and noticing than $(1-x)^{-a} L_{n}(w, v, x)$ has a zero at 1 with multiplicity at least $-a$. Proceeding this way we obtain (96)

$$
L_{n}(w, v, x)-v(x)=\left.(1-x)^{a} p_{n}(w, x) \sum_{l=0}^{-a-1}(-1)^{l+1} \frac{1}{l !}\left[\frac{(1+t)^{b}}{p_{n}(w, t)}\right]^{(l)}\right|_{t=1}(1-x)^{l} .
$$

If $w$ is given by (10) then, since $v \sqrt{w \sqrt{1-x^{2}}} \in L^{1}$, we have $\delta=2 a+\Gamma_{0}+5 / 2>0$. Let $\varepsilon$ be chosen so that $t_{1}<\varepsilon<1$ where $t_{1}$ is defined in (10). Since $a \leqslant-1, \Gamma_{0}$ must be greater than $-1 / 2$ and thus by (15),

$$
\left|p_{n}(w, x)\right|(1-x)^{l} \leqslant \text { const }(1-x)^{l} / \sqrt{w(x) \sqrt{1-x^{2}}}, \quad \varepsilon \leqslant x \leqslant 1,
$$

$l=0,1, \ldots, a-1$. Applying (26) we obtain

$$
\begin{array}{r}
\left|p_{n}(w, x)\right|(1-x)^{l} \leqslant \text { const } n^{-2 l} / \sqrt{w\left(1-n^{-2}\right) \sqrt{n^{-2}}} \leqslant \text { const } n^{-2 l+\Gamma_{0}+1 / 2}, \\
\varepsilon \leqslant x \leqslant 1,
\end{array}
$$

$l=0,1, \ldots,-a-1$. It follows from (17) and (23) that

$$
\left|\left[\frac{(1+t)^{b}}{p_{n}(w, t)}\right]^{(l)}\right|_{t=1} \mid \leqslant \text { const } n^{2 l-\Gamma_{0}-1 / 2}
$$

for $l=0,1, \ldots,-a-1$. Combining (96)-(98) we get

$$
\left|L_{n}(w, v, x)-v(x)\right| \leqslant \text { const } v(x), \quad \varepsilon \leqslant x \leqslant 1 .
$$

For $-1 \leqslant x \leqslant \varepsilon$ we can apply (16), (96) and (98) to obtain

$$
\left|L_{n}(w, v, x)-v(x)\right| \leqslant \mathrm{const}\left[1 / \sqrt{w(x) \sqrt{1-x^{2}}}+1\right] n^{-\delta}, \quad-1 \leqslant x \leqslant \varepsilon,
$$

where $\delta>0$. Now (95) follows immediately from (99) and (100). If $a>0$ and $b \leqslant 0$ then we can use similar arguments to prove (95). If both $a$ and $b$ are negative, then $v^{-1}\left[L_{n}(w, v)-v\right]$ is a polynomial of degree $-a-b+n-1$ which vanishes at zeros 
of $p_{n}(w)$. Using the Hermite interpolation formula we obtain (101)

$$
\begin{aligned}
L_{n}(w, v, x)-v(x)= & \left.(1-x)^{a} p_{n}(w, x) \sum_{l=0}^{-a-1}(-1)^{l+1} \frac{1}{l !}\left[\frac{(1+t)^{b}}{p_{n}(w, t)}\right]^{(l)}\right|_{t=1}(1-x)^{l} \\
& -(1+x)^{l} p_{n}(w, x) \sum_{l=0}^{-b-1} \frac{1}{l !}\left[\left.\left.\frac{(1-t)^{a}}{p_{n}(w, t)}\right|^{(l)}\right|_{t=-1}(1+x)^{l} .\right.
\end{aligned}
$$

Choosing $\varepsilon$ so that $t_{1}<\varepsilon<1$ and $-1<-\varepsilon<t_{m}$ (see (10)) we get that both (97) and (98) hold and by similar arguments we obtain

$$
\left|p_{n}(w, x)\right|(1+x)^{l} \leqslant \text { const } n^{-2 l+\Gamma_{m+1}+1 / 2}, \quad-1 \leqslant x \leqslant-\varepsilon,
$$

and

$$
\left|\left[\frac{(1-t)^{a}}{p_{n}(w, t)}\right]^{(l)}\right|_{t=-1} \mid \leqslant \text { const } n^{2 l-\Gamma_{m+1}-1 / 2}, \quad-1 \leqslant x \leqslant-\varepsilon,
$$

for $l=0,1, \ldots,-b-1$. Since $v \sqrt{w \sqrt{1-x^{2}}} \in L^{1}$ we get that both $\delta=2 a+\Gamma_{0}+$ $5 / 2$ and $\delta_{1}=2 b+\Gamma_{m+1}+5 / 2$ are positive. Now from (97), (98) and (101)-(103) we obtain

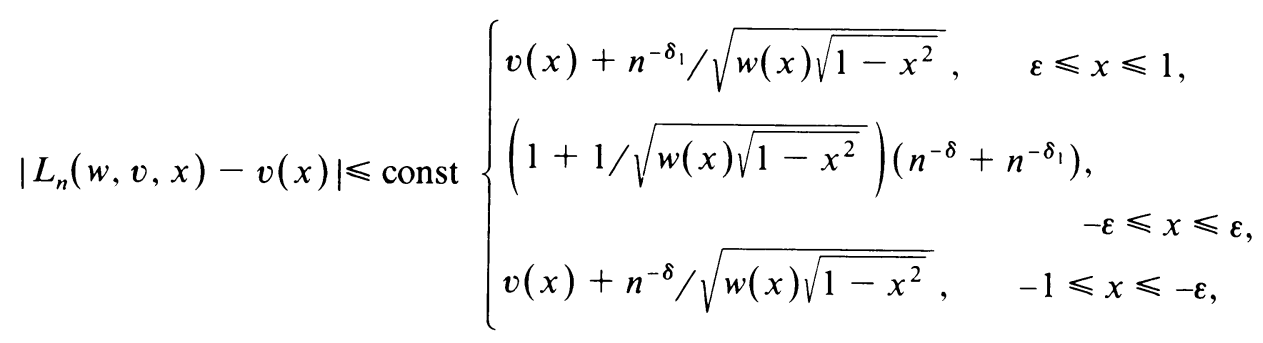

and the theorem follows again.

THEOREM 5. Let $w \in G S J, 0<p<\infty$ and let $v$ be a not necessarily integrable Jacobi weight, say $v(x)=(1-x)^{a}(1+x)^{b}$ for $|x| \leqslant 1$. Let $v^{*}$ be a rational Jacobi weight defined by $v^{*}(x)=(1-x)^{[a]}(1+x)^{[b]}$ for $|x| \leqslant 1$. If $u$ is a nonnegative function supported in $[-1,1]$ and $u \in L^{p}, u v^{*} \in\left(L \log ^{+} L\right)^{p}, u / \sqrt{w \sqrt{1-x^{2}}} \in L^{p}$ and $v^{*} \sqrt{w \sqrt{1-x^{2}}} \in L^{1}$ then

$$
\lim _{n \rightarrow \infty}\left\|\left[L_{n}(w, v f)-v f\right] u\right\|_{p}=0
$$

holds for every continuous function $f$ in $[-1,1]$.

Proof. By Theorems 3 and 4 we have

$$
\lim _{n \rightarrow \infty}\left\|\left[L_{n}\left(w, v^{*} F\right)-v^{*} F\right] u\right\|=0
$$

for every $F \in C[-1,1]$ and by the construction of $v^{*}$ the function $F=v\left(v^{*}\right)^{-1} f$ is continuous whenever $f$ is continuous. Thus (104) holds by (105). 
THEOREM 6. Let $w \in G S J, 0<p<\infty$, and let $r$ and $s$ be nonnegative integers. Let $u$ be a nonnegative function defined in $[-1,1]$ such that $u \in\left(L \log ^{+} L\right)^{p}$ and $u$ is positive on a set with positive measure. Let $L_{n}^{(r, s)}(w, f)$ be the quasi-Lagrange interpolating polynomial defined by (5)-(8) and let $v(x)=(1-x)^{-r}(1+x)^{-s}$. Then

$$
\lim \left\|\left[L_{n}^{(r, s)}(w, f)-f\right] u\right\|_{p}=0, \quad \forall f \in C[-1,1],
$$

holds if and only if

$$
v \sqrt{w \sqrt{1-x^{2}}} \in L^{1} \quad \text { and } \quad u /\left(v \sqrt{w \sqrt{1-x^{2}}}\right) \in L^{p}
$$

Moreover, there exists a nonnegative $u$ such that $u \in L^{p} \backslash\left(L \log ^{+} L^{p}\right)$ and (107) does not imply (106).

Proof. We start with writing $L_{n}^{(r, s)}(w, f)$ in the form

$$
L_{n}^{(r, s)}(w, f)=v^{-1} L_{n}(w, v f)+f(1) Q+f(-1) R
$$

where

$$
Q(x)=\left.(1+x)^{s} p_{n}(w, x) \sum_{l=0}^{r-1}(-1)^{l} \frac{1}{l !}\left[\frac{1}{p_{n}(w, t)(1+t)^{s}}\right]^{(1)}\right|_{t=1}(1-x)^{l}
$$

and

$$
R(x)=\left.(1-x)^{r} p_{n}(w, x) \sum_{l=0}^{s-1} \frac{1}{l !}\left[\frac{1}{p_{n}(w, t)(1-t)^{r}}\right]^{(l)}\right|_{t=-1}(1+x)^{l}
$$

If either $r$ or $s$ equals 0 then the corresponding polynomial $Q$ or $R$ is identically 0 . (108)-(110) follow immediately from (5)-(8). If (106) holds then by the uniform boundedness principle [13, p. 182]

$$
\sup _{n \geqslant 1}\left\|L_{n}^{(r, s)}(w, f) u\right\|_{p} \leqslant \text { const }\|f\|_{\infty}
$$

for every $f \in C[-1,1]$, in particular, if $f( \pm 1)=0$. Thus by (108)

$$
\sup _{n \geq 1}\left\|L_{n}(w, v f) v^{-1} u\right\|_{p} \leqslant \text { const }\|f\|_{\infty}
$$

for every continuous function $f$ vanishing at \pm 1 . Applying Theorem 2 we see that conditions (107) are satisfied. Conversely, if (107) holds then by Theorem 5

$$
\lim _{n \rightarrow \infty}\left\|\left[L_{n}(w, v F)-v F\right] v^{-1} u\right\|_{p}=0
$$

for every $F \in C[-1,1]$. For $f \in C[-1,1]$ let $P$ be any fixed polynomial satisfying

$$
\left.P(t)^{(j)}\right|_{t=1}=f(1) \delta_{0 j}, \quad 0 \leqslant j \leqslant r-1,
$$

and

$$
\left.P(t)^{(j)}\right|_{t=-1} f(-1) \delta_{0 j}, \quad 0 \leqslant j \leqslant s-1 .
$$

If $r+s+n-1 \geqslant \operatorname{deg} P$ then

$$
S_{n}=v^{-1} L_{n}(w,(f-P) v)+P-L_{n}^{(r, s)}(w, f)
$$


is a polynomial of degree $r+s+n-1$ satisfying

$$
\begin{array}{ll}
S_{n}\left(x_{k n}(w)\right)=0, & 1 \leqslant k \leqslant n, \\
\left.S_{n}(t)^{(j)}\right|_{t=1}=0, & 0 \leqslant j \leqslant r-1,
\end{array}
$$

and

$$
\left.S_{n}(t)^{(j)}\right|_{t=-1}=0, \quad 0 \leqslant j \leqslant s-1,
$$

so that $S_{n}$ is identically 0 . Thus

$$
L_{n}^{(r . s)}(w, f)=v^{-1} L_{n}(w,(f-P) v)+P
$$

and if $F$ is defined by $F=f-P$ then

$$
L_{n}^{(r, s)}(w, f)-f=v^{-1}\left[L_{n}(w, v F)-v F\right] .
$$

Hence by (112), (106) is true for every $f \in C[-1,1]$. Now we turn to the last part of the theorem. Suppose that for every nonnegative $u \in L^{p}$ satisfying (107), (106) holds. Then (111) holds as well, and by (108)

$$
\sup _{n \geq 1}\left\|L_{n}(w, v f) v^{-1} u\right\|_{p} \leqslant \text { const }\|f\|_{x}
$$

is satisfied for every continuous function $f$ vanishing at \pm 1 . But according to Theorem 2, (113) fails for some $u \in L^{p}$ and some $f \in C[-1,1]$ with $f( \pm 1)=0$. This shows that (106) cannot be true for every $u \in L^{p}$ when (107) holds.

Partial cases of Theorem 6 have been proved in $[1,2,5,6,10,11,14$ and 19].

Even though it is unlikely that $u v$ must belong to $\left(L \log ^{+} L\right)^{p}$ whenever (73) holds for every continuous function $f$, the following theorem demonstrates that this, in fact, is not too far from the truth.

THEOREM 7. Let $w \in G S J$ and $0<p<\infty$. Suppose that either

$$
0<\lim _{x \rightarrow 1} w(x) \sqrt{1-x^{2}}<\infty
$$

or

$$
0<\lim _{x \rightarrow-1} w(x) \sqrt{1-x^{2}}<\infty
$$

holds. Then there exists a nonnegative function $u$ with support in $[-1,1]$ such that $u / \sqrt{w \sqrt{1-x^{2}}} \in L^{p}$ and $u \in L^{p}\left(\log ^{+} L\right)^{q}$ for every $q<p$ and nevertheless

$$
\sup _{n \geqslant 1} \sup _{\substack{n f \|_{\infty} \leqslant 1 \\ f \in C}}\left\|L_{n}(w, f) u\right\|_{p}=\infty .
$$

Proof. Assume without loss of generality that (114) holds. Pick $\varepsilon$ such that $0<\varepsilon<1$ and

$$
w(x) \sqrt{1-x^{2}} \sim 1, \quad \varepsilon \leqslant x \leqslant 1
$$

is satisfied. Let $1_{\varepsilon}$ be the characteristic function of $[\varepsilon, 1]$. Define $u$ by

$$
u(x)=\left.1_{\varepsilon}(x)(1-x)^{-1 / p}|\log (1-x)|^{-1-1 / p}|\log | \log (1-x)\right|^{1 / p} .
$$


Then $u \in L^{p}$ so that by (117) $u / \sqrt{w \sqrt{1-x^{2}}} \in L^{p}$ as well. If $q<p$ then, since

$$
\left(\log ^{+} u(x)\right)^{q} \leqslant \text { const } 1_{\varepsilon}(x)|\log (1-x)|^{q}, \quad|x| \leqslant 1,
$$

we have

$$
u(x)^{p}\left(\log ^{+} u(x)\right)^{q} \leqslant \text { const } 1_{\varepsilon}(x)(1-x)^{-1}|\log (1-x)|^{q-p-1}|\log | \log (1-x)||
$$

and hence $u \in L^{p}\left(\log ^{+} L\right)^{q}$. Now choose $c>0$ such that $1-c n^{-2}>\frac{1}{2}\left(1+x_{1 n}(w)\right)$ holds for every $n \in \mathbf{N}$. This can be accomplished by (11). If $n$ is large enough then

$$
\begin{aligned}
\int_{1-c n^{-2}}^{1}|u(x)|^{p} d x & \geqslant \text { const } \log \log n \int_{1-c n^{-2}}^{1}(1-x)^{-1}|\log (1-x)|^{-p-1} d x \\
& \geqslant \text { const } \log \log n \cdot(\log n)^{-p}
\end{aligned}
$$

and consequently

$$
\sup _{n \geqslant 1}(\log n)^{p} \int_{1-n^{-2}}^{1}|u(x)|^{p} d x=\infty .
$$

For every $n$ pick a continuous function $f_{n}$ such that $f_{n}\left(x_{k n}\right)=\operatorname{sign} p_{n-1}\left(w, x_{k n}\right)$ for $\varepsilon \leqslant x_{k n}<1, f_{n}\left(x_{k n}\right)=0$ for $x_{k n}<\varepsilon$ and $\left|f_{n}(x)\right| \leqslant 1$ for $|x| \leqslant 1$. Then

$$
L_{n}\left(w, f_{n}, x\right)=\sum_{x_{k n}>\varepsilon}\left|l_{k n}(w, x)\right|, \quad 1-c n^{-2} \leqslant x \leqslant 1,
$$

and by (3), (11), (14), (20) and (21)

$$
L_{n}\left(w, f_{n}, x\right) \geqslant \text { const } \log n p_{n}(w, x), \quad 1-c n^{-2} \leqslant x \leqslant 1 .
$$

The next step is to show that

$$
p_{n}(w, x) \geqslant \text { const, } \quad 1-c n^{-2} \leqslant x \leqslant 1 .
$$

We have

$$
\begin{array}{r}
\frac{p_{n}(w, x)}{p_{n}(w, 1)}=\prod_{k=1}^{n} \frac{x-x_{k n}}{1-x_{k n}}=\prod_{k=1}^{n}\left(1-\frac{1-x}{1-x_{k n}}\right) \geqslant \prod_{k=1}^{n}\left(1-\frac{1-x_{1 n}}{2\left(1-x_{k n}\right)}\right), \\
1-c n^{-2} \leqslant x \leqslant 1,
\end{array}
$$

since $1-c n^{-2} \geqslant \frac{1}{2}\left(1+x_{1 n}\right)$. But $\log (1-x) \geqslant-2 x$ for $0 \leqslant x \leqslant \frac{1}{2}$. Thus

$$
\begin{aligned}
\frac{p_{n}(w, x)}{p_{n}(w, 1)} & \geqslant \exp \left[-\left(1-x_{1 n}\right) \sum_{k=1}^{n} \frac{1}{1-x_{k n}}\right] \\
& =\exp \left[-\left(1-x_{1 n}\right) p_{n}(w, 1)^{\prime} p_{n}(w, 1)^{-1}\right], \quad 1-c n^{-2} \leqslant x \leqslant 1 .
\end{aligned}
$$

Now (121) follows from (11), (17) and (22). We obtain from (120) and (121) that

$$
\int_{1-c n^{-2}}^{1}\left|L_{n}\left(w, f_{n}\right) u\right|^{p} \geqslant \operatorname{const}(\log n)^{p} \int_{1-c n^{-2}}^{1}|u|^{p}
$$

and by (119) we see that (116) holds when $u$ is defined by (118).

\section{REFERENCES}

1. R. Askey, Mean convergence of orthogonal series and Lagrange interpolation, Acta Math. Acad. Sci. Hungar. 23 (1972), 71-85.

2. , Summability of Jacobi series, Trans. Amer. Math. Soc. 179 (1973), 71-84.

3. V. Badkov, Convergence in the mean and almost everywhere of Fourier series in polynomials orthogonal on an interval, Math. USSR-Sb. 24 (1974), 223-256. 
4. N. Dunford and J. T. Schwartz, Linear operators, Vol. 2, Interscience, New York, 1958.

5. P. Erdös and E. Feldheim, Sur le mode de convergence pour l'interpolation de Lagrange, C. R. Acad. Sci. Paris Ser. A-B 203 (1936), 913-915.

6. P. Erdös and P. Turán, On interpolation. I, II, III. Ann. of Math. 38 (1937), 142-151; 39 (1938), $703-724 ; 41$ (1940), 510-553.

7. G. Freud, Orthogonal polynomials, Pergamon Press, New York, 1971.

8. Ü Üher eine Klasse Lagrangescher Interpolationsverfahren, Studia Sci. Math. Hungar. 3 (1968), 249-255.

9. E. Hille and R. S. Phillips, Functional analysis and semigroups, Amer. Math. Soc. Colloq. Publ., vol. 31, Amer. Math. Soc., Providence, R. I., 1957.

10. M. Kuetz, A note on mean convergence of Lagrange interpolation, J. Approx. Theory 35 (1982), $77-82$.

11. J. Marcinkiewicz, Sur l'interpolation. I, Studia Math. 6(1936), 1-17.

12. I. P. Natanson, Constructive function theory, Vol. 1, Ungar, New York, 1964.

13. P. Nevai, Orthogonal polynomials, Mem. Amer. Math. Soc. no. 213, 1979.

14. Mean convergence of Lagrange interpolation. I, II, J. Approx. Theory 18 (1976), 363-377: 30 (1980), 263-276.

15. Lagrange interpolation at zeros of orthogonal polynomials, Approximation Theory. II (G. G. Lorentz et al., eds.), Academic Press, New York, 1976, pp. 163-201.

16. P. Nevai and P. Vértesi, Mean convergence of Hermite-Fejér interpolation, J. Math. Anal. Appl. (to appear).

17. E. Stein, Singular integrals and differentiability properties of functions, Princeton Univ. Press, Princeton, N. J., 1970.

18. G. Szegö, Orthogonal polynomials, Amer. Math. Soc. Colloq. Publ., vol. 23. Amer. Math. Soc., Providence, R. I., 1967.

19. A. K. Varma and P. Vértesi, some Erdös-Feldheim type theorems on mean convergence of Lagrange interpolation, J. Math. Anal. Appl. 91 (1983), 68-79.

20. P. Vértesi, Remarks on the Lagrange interpolation, Studia Sci. Math. Hungar. 15 (1980), 277-281.

Department of Mathematics, Ohio State University, Columbus, Ohio 43210 